\title{
Energy Contribution of a Point-Interacting Impurity in a Fermi Gas
}

\author{
Thomas Moser and Robert Seiringer
}

\begin{abstract}
We give a bound on the ground-state energy of a system of $N$ non-interacting fermions in a three-dimensional cubic box interacting with an impurity particle via point interactions. We show that the change in energy compared to the system in the absence of the impurity is bounded in terms of the gas density and the scattering length of the interaction, independently of $N$. Our bound holds as long as the ratio of the mass of the impurity to the one of the gas particles is larger than a critical value $m^{* *} \approx 0.36$, which is the same regime for which we recently showed stability of the system.
\end{abstract}

\section{Introduction}

Quantum systems of particles interacting with forces of very short range allow for an idealized description in terms of point interactions. The latter are characterized by a single number, the scattering length. Originally point interactions were introduced in the 1930s to model nuclear interactions $[4,5,12,28,29]$, but later they were also successfully applied to many other areas of physics, like polarons (see [20] and references there) or cold atomic gases [30].

It was already known to Thomas [28] that the spectrum of a bosonic many-particle system depends strongly on the range of the interactions, and that an idealized point-interacting system with more than two particles is inherently unstable, i.e., the energy is not bounded from below. This collapse can be counteracted by the Pauli principle for fermions with two species (e.g., spin states). In this paper, we are interested in the impurity problem where there is only one particle for one of the species.

Given $N \geq 1$ fermions of one type with mass 1 and one particle of another type with mass $m>0$, a model of point interactions gives a meaning to the formal expression

$$
-\frac{1}{2 m} \Delta_{y}-\frac{1}{2} \sum_{i=1}^{N} \Delta_{x_{i}}+\gamma \sum_{i=1}^{N} \delta\left(x_{i}-y\right)
$$


for $\gamma \in \mathbb{R}$. We note that because of the antisymmetry constraint on the wavefunctions there are only interactions between particles of different species. Expression (1.1) is ill-defined in $d \geq 2$ dimensions since $H^{1}\left(\mathbb{R}^{d}\right)$, the form domain of the Laplacian, contains discontinuous functions for which the meaning of the $\delta$-function as a potential is unclear. In the following, we restrict our attention to the case $d=3$, but we note that also two-dimensional systems exhibit interesting behavior $[10,11,15,16,18,19]$. For $d \geq 4$, there are no point interactions as the Laplacian restricted to functions supported away from the hyperplanes of interactions is essentially self-adjoint.

A mathematically precise meaning to (1.1) in three dimensions was given in $[10,13,21]$, and we will work with the model introduced there. Our analysis will start from this well-defined model, but we note that the question whether the model can be obtained as a limit of Schrödinger operators with genuine interaction potentials of shrinking support is still open. (See, however, [1] for the case $N=1$, and [2] for models in one dimension.)

In this paper, we study the energy contribution of the point-interacting impurity. We confine the $N+1$ particles to a box $(0, L)^{3}$ and investigate the ground-state energy of the system. In particular, our goal is to show that at given mean particle density $\bar{\rho}=N / L^{3}$, the difference between the groundstate energies of the interacting and the non-interacting system is bounded independently of the system size.

Previous work on this model was mostly concerned with stability and hence studied the model without confinement. For example, it is possible to analyze the $2+1$ model, i.e., two fermions of one kind and one impurity of another kind, in great detail [3,6-8,10,21-24]. It turns out that the mass of the impurity plays an important role for stability. It was shown in [6] that for the $2+1$ system there is a critical mass $m^{*} \approx 0.0735$ such that the system is stable for $m \geq m^{*}$ and unstable otherwise. This critical mass does not depend on the strength of the interaction, i.e., the scattering length.

Building on these results, it was shown in [25] that a similar statement holds for the $N+1$ system. In particular, it was proven that there is a critical mass $m^{* *} \approx 0.36$ such that the system is stable for all $m \geq m^{* *}$, independently of $N$. This bound is presumably not sharp, and stability is still open for $m \in$ $\left[m^{*}, m^{* *}\right)$. Recently also the stability of the $2+2$ system was proved in a suitable mass range [26]. The general case with $N+M$ particles still poses an open problem, however.

In all cases where stability of the system was established, the groundstate energy in infinite volume is actually zero in case the scattering length is negative, and there are no bound states. For positive scattering length there are bound states, but one still expects that only a finite number of particles can bind to the impurity. In particular, the ground-state energy of the $N+1$ system is bounded from below independently of $N$ [25]. Intuitively, one would expect that if one confines the system to a box in order to have a nonzero mean particle density, the interaction with the impurity should again only affect a finite number of particles, and hence the energy change compared to the noninteracting system should be $O(1)$, independently of $N$. This is what we prove 
here. We note that it is sufficient to derive a lower bound on the ground-state energy, as point interactions are always attractive, i.e., they lower the energy.

Even for regular interaction potentials, it is highly non-trivial to show that an impurity causes only an $O(1)$ change to the energy of a non-interacting Fermi gas. For fixed, i.e., non-dynamical impurities, this was established in [14] as a consequence of a positive density version of the Lieb-Thirring inequality. The result in [14] applies to systems in infinite volume, as well as to systems in a box with periodic boundary conditions. In the appendix, we provide an extension to Dirichlet boundary conditions, since this result will be an essential ingredient in our proof.

Compared to [14], we face here two additional difficulties: the impurity is dynamic and has a finite mass, and the interaction with the gas particles is through singular point interactions. Besides the methods of [14] and [25], a key ingredient in our analysis is a proof of an IMS type formula for the quadratic form defining the model, which allows for a localization of the particles into regions close and far away from the impurity. It has the same form as the IMS formula for regular Schrödinger operators (see [9, Theorem 3.2]), but is much harder to prove.

\subsection{The Point Interaction Model}

We consider a system of $N$ fermions of mass 1 , interacting with another particle of mass $m>0$. Let

$$
H_{0}^{N}=-\frac{1}{2 m} \Delta_{0}-\frac{1}{2} \sum_{i=1}^{N} \Delta_{i}
$$

be the non-interacting part of the Hamiltonian, acting on $L^{2}\left(\mathbb{R}^{3}\right) \otimes L_{\text {as }}^{2}\left(\mathbb{R}^{3 N}\right)$, where $L_{\text {as }}^{2}$ denotes the totally antisymmetric functions in $\otimes^{N} L^{2}\left(\mathbb{R}^{3}\right)$. The $N+$ 1 coordinates we denote by $x_{0}, x_{1}, \ldots, x_{N} \in \mathbb{R}^{3}$ and throughout this paper we will use the notation $\vec{x}=\left(x_{1}, \ldots, x_{N}\right)$. If we want to exclude a set of coordinates labeled by $A \subseteq\{1, \ldots, N\}$ we use $\hat{x}_{A}=\left(x_{i}\right)_{i \notin A}$ and for short $\hat{x}_{i}=\hat{x}_{\{i\}}$. If we want to restrict to certain coordinates we write $\vec{x}_{A}=\left(x_{i}\right)_{i \in A}$.

For $\mu>0$, we define $G_{\mu}$ as the resolvent of $H_{0}^{N}$ in momentum space, i.e.,

$$
G_{\mu}\left(k_{0}, \vec{k}\right):=\left(\frac{1}{2 m} k_{0}^{2}+\frac{1}{2} \vec{k}^{2}+\mu\right)^{-1} .
$$

We denote by $F_{\alpha, N}$ the quadratic form used in [6,25] describing point interactions between $N$ fermions and the impurity. Its domain is given by

$$
\begin{array}{r}
D\left(F_{\alpha, N}\right)=\left\{\psi=\phi_{\mu}+G_{\mu} \xi \mid \phi_{\mu} \in H^{1}\left(\mathbb{R}^{3}\right) \otimes H_{\mathrm{as}}^{1}\left(\mathbb{R}^{3 N}\right),\right. \\
\left.\xi \in H^{1 / 2}\left(\mathbb{R}^{3}\right) \otimes H_{\mathrm{as}}^{1 / 2}\left(\mathbb{R}^{3(N-1)}\right)\right\}
\end{array}
$$

where $G_{\mu} \xi$ is defined via its Fourier transform (denoted by a $\hat{\bullet}$ ) as

$$
\widehat{G_{\mu} \xi}\left(k_{0}, \vec{k}\right)=G_{\mu}\left(k_{0}, \vec{k}\right) \sum_{i=1}^{N}(-1)^{i+1} \hat{\xi}\left(k_{0}+k_{i}, \hat{k}_{i}\right) .
$$

The space $H_{\text {as }}^{1}\left(\mathbb{R}^{3 N}\right)$ contains all totally antisymmetric functions in $H^{1}\left(\mathbb{R}^{3 N}\right)$. For a given $\psi \in D\left(F_{\alpha, N}\right)$ and $\mu>0$, the splitting $\psi=\phi_{\mu}+G_{\mu} \xi$ is unique. We 
point out that while $\phi_{\mu}$ depends on the choice of $\mu, \xi$ is independent of $\mu$. We will call $\phi_{\mu}$ the regular part and $\xi$ the singular part of $\psi$. Note that $D\left(F_{\alpha, N}\right)$ is independent of the choice of $\mu$, and so is the quadratic form $F_{\alpha, N}$ defined as

$$
\begin{aligned}
F_{\alpha, N}(\psi) & :=\left\langle\phi_{\mu}\left|H_{0}^{N}+\mu\right| \phi_{\mu}\right\rangle-\mu\|\psi\|_{L^{2}\left(\mathbb{R}^{3(N+1)}\right)}^{2}+T_{\alpha, \mu, N}(\xi) \\
T_{\alpha, \mu, N}(\xi) & :=N\left(\frac{2 m}{m+1} \alpha\|\xi\|_{L^{2}\left(\mathbb{R}^{3 N}\right)}^{2}+T_{\text {dia }}^{\mu, N}(\xi)+T_{\text {off }}^{\mu, N}(\xi)\right)
\end{aligned}
$$

where

$$
\begin{aligned}
& T_{\text {dia }}^{\mu, N}(\xi):=\int_{\mathbb{R}^{3 N}}|\hat{\xi}(\vec{k})|^{2} L_{\mu, N}(\vec{k}) \mathrm{d} \vec{k} \\
& T_{\text {off }}^{\mu, N}(\xi):=(N-1) \int_{\mathbb{R}^{3(N+1)}} \hat{\xi}^{*}\left(k_{0}+k_{1}, \hat{k}_{1}\right) \hat{\xi}\left(k_{0}+k_{2}, \hat{k}_{2}\right) G_{\mu}\left(k_{0}, \vec{k}\right) \mathrm{d} k_{0} \mathrm{~d} \vec{k} \\
& L_{\mu, N}(\vec{k}):=2 \pi^{2}\left(\frac{2 m}{m+1}\right)^{3 / 2}\left(\frac{k_{1}^{2}}{2(m+1)}+\frac{1}{2} \hat{k}_{1}^{2}+\mu\right)^{1 / 2}
\end{aligned}
$$

The quadratic form $F_{\alpha, N}$ describes $N$ fermions interacting with an impurity particle via point interactions with scattering length $a=-2 \pi^{2} / \alpha$, with $\alpha \in \mathbb{R}$. The non-interacting system is recovered in the limit $\alpha \rightarrow+\infty$.

Notation. Throughout the paper, we will use the following notation. We define the relation $\lesssim$ by

$$
x \lesssim y \Longleftrightarrow \exists C>0: x \leq C y
$$

where $C$ is independent of $x$ and $y$. In the obvious way, we define $\gtrsim$. In case that $x \lesssim y$ and $y \lesssim x$ we write $x \sim y$.

\section{Main Result for Confined Wavefunctions}

Let us assume that $\operatorname{supp} \psi \subset \mathcal{C}_{L}^{N+1}$, where $\mathcal{C}_{L}=(0, L)^{3}$ for some $L>0$. The mean particle density will be denoted by $\bar{\rho}=N / L^{3}$. Let $E_{N}^{D}$ be the ground-state energy of $-\frac{1}{2} \sum_{i=1}^{N} \Delta_{i}$ for wavefunctions in $H_{\text {as }}^{1}\left(\mathbb{R}^{3 N}\right)$ with Dirichlet boundary conditions on $\partial \mathcal{C}_{L}$. It equals the sum of the $N$ lowest eigenvalues of the Dirichlet Laplacian on $\mathcal{C}_{L}$, and it is easy to see that

$$
E_{N}^{D} \sim N \bar{\rho}^{2 / 3}
$$

A natural question is how the interactions affect this energy. From [25, Theorem 2.1], we know that there is a mass-dependent constant $\Lambda(m)$ [25, Eq. (2.8)], given in Eq. (4.53) below, such that if $\Lambda(m)<1$ then $F_{\alpha, N}$ is bounded from below independently of $N$ by

$$
\frac{F_{\alpha, N}(\psi)}{\|\psi\|_{2}^{2}} \geq \frac{m+1}{2 m} \begin{cases}0 & \alpha \geq 0 \\ -\left(\frac{\alpha}{2 \pi^{2}(1-\Lambda(m))}\right)^{2} & \text { otherwise. }\end{cases}
$$


(The additional factor $(m+1) /(2 m)$ compared to [25, Theorem 2.1] results from the separation of the center-of-mass motion used in [25].) It was also shown in [25] that $\Lambda(m)<1$ if $m>m^{* *} \approx 0.36$.

For particles confined to the cube $\mathcal{C}_{L}$ with mean density $\bar{\rho}$, we can show that under the condition $\Lambda(m)<1$ the correction to $E_{N}^{D}$ is small, i.e., it is $O(1)$ independently of $N$. Our main result is the following.

Theorem 2.1. Let $\psi \in D\left(F_{\alpha, N}\right)$, supported in $(0, L)^{3(N+1)}$, with $\|\psi\|=1$. Let $\bar{\rho}=N L^{-3}$, and assume that $\Lambda(m)<1$. Then

$$
F_{\alpha, N}(\psi) \geq E_{N}^{D}-\text { const. }\left(\frac{\bar{\rho}^{2 / 3}}{(1-\Lambda(m))^{9 / 2}}+\frac{\alpha_{-}^{2}}{(1-\Lambda(m))^{2}}\right)
$$

where the constant is independent of $\psi, m, N, L$ and $\alpha$, and $\alpha_{-}$denotes the negative part of $\alpha$, i.e., $\alpha_{-}=\frac{1}{2}(|\alpha|-\alpha)$.

Theorem 2.1 shows that the presence of the impurity affects the groundstate energy by a term that is bounded independently of $N$. Bound (2.3) is an extension of (2.2) in the sense that if we take $L \rightarrow \infty$ in (2.3) we recover (2.2) up to the value of the constant.

Remark. For $\alpha \rightarrow \infty$, one would expect that the optimal lower bound converges to the ground-state energy of the non-interacting Hamiltonian $H_{0}^{N}$ with Dirichlet boundary conditions. This is not the case for (2.3), which is independent of $\alpha$ for $\alpha \geq 0$. This is due to our method of proof; in particular, the utilized localization technique introduces an error which is independent of the interaction strength.

Using various types of trial states, the ground-state energy of pointinteracting systems is extensively discussed in the physics literature (see [20] and references there). We note that with this method it is only possible to derive upper bounds, while Theorem 2.1 gives a lower bound on the groundstate energy.

From a physics perspective, it would of course be interesting to extend Theorem 2.1 to the case of several (or even many) impurities [30]. However, even the basic question of stability, i.e., boundedness of the energy from below, is open for more than one impurity.

\subsection{Proof Outline}

For the proof of Theorem 2.1, we first prove in Sect. 3 an IMS type formula, which allows to localize the impurity in a small box, of side length $\ell$ independent of $L$. In a second step, we localize all of the remaining particles to be either close to the impurity or separated from it. Doing this, we partly violate the antisymmetry constraint on the wavefunctions, which makes it necessary to first extend the quadratic form $F_{\alpha, N}$ to $\tilde{F}_{\alpha, N}$. The latter does not require the antisymmetry, but coincides with $F_{\alpha, N}$ on $D\left(F_{\alpha, N}\right)$.

In Sect. 4 , we give a rough lower bound on the energy in case the wavefunction is compactly supported in a box $(0, \ell)^{3}$. This lower bound is of the order $N^{5 / 3} / \ell^{2}$, as expected, but with a non-sharp prefactor. We shall introduce 
a quadratic form $F_{\alpha, N}^{\text {per }}$ with periodic boundary conditions and show that it is equivalent to $F_{\alpha, N}$ for confined wavefunctions. The reason we work with periodic boundary conditions instead of Dirichlet ones is that the simpler form of the Greens function for the Laplacian allows to perform explicit computations in momentum space.

Because the ground-state energy of the confined non-interacting $N$ particle system is strictly positive, we are allowed to choose $\mu$ negative in the definition of $F_{\alpha, N}^{\text {per }}$. Applying the method of [25] then leads to the lower bound on $F_{\alpha, N}^{\text {per }}$ in Theorem 4.1. The downside of working with $F_{\alpha, N}^{\text {per }}$ will be that because of the discrete nature of momentum space for periodic functions, we have to work with sums instead of integrals, and the difference between the sum and the integral versions will have to be carefully controlled.

In Sect. 5, we give the proof of Theorem 2.1. Using the IMS formula of Proposition 3.1, we localize the particles either in a small box with side length $\ell \sim \bar{\rho}^{-1 / 3}$ containing the impurity, or in the large complement. In the small box, we use Theorem 4.1 for a lower bound, whereas in the large complement we use Theorem A.2, which is a version of the positive density Lieb-Thirring inequality in [14] adapted to our setting of Dirichlet boundary conditions, and which is proved in the appendix. When optimizing over the distribution of particles, we shall conclude from these bounds that only $O\left(\bar{\rho} \ell^{3}\right)=O(1)$ particles will be in the small box. This allows us to improve the rough bound of Theorem 4.1 and show Theorem 2.1.

\section{Properties of the Quadratic Form}

In this section, we will first extend the quadratic form $F_{\alpha, N}$ to functions that are not required to be antisymmetric in the last $N$ variables. Afterward, we shall discuss how the splitting $\psi=\phi_{\mu}+G_{\mu} \xi$ is affected when multiplying $\psi$ by a smooth function (which need not be symmetric under permutations). This will be utilized in the last part of this section where an IMS formula for the (extended) quadratic form is shown.

\subsection{Extension to Functions Without Symmetry}

To prove our main theorem, we want to localize the particles in different subsets of the cube $\mathcal{C}_{L}=(0, L)^{3}$. Hence, it is necessary to extend the quadratic form $F_{\alpha, N}$ by removing the antisymmetry constraint. To this aim, we define

$$
\begin{aligned}
D\left(\tilde{F}_{\alpha, N}\right)=\left\{\psi=\phi_{\mu}+\sum_{i=1}^{N} G_{\mu} \xi_{i} \mid \phi_{\mu}\right. & \in H^{1}\left(\mathbb{R}^{3(N+1)}\right), \\
\xi_{i} & \left.\in H^{1 / 2}\left(\mathbb{R}^{3 N}\right) \forall i, 1 \leq i \leq N\right\}
\end{aligned}
$$

where

$$
\widehat{G_{\mu} \xi_{i}}\left(k_{0}, \vec{k}\right)=G_{\mu}\left(k_{0}, \vec{k}\right) \hat{\xi}_{i}\left(k_{0}+k_{i}, \hat{k}_{i}\right) .
$$


The quadratic form $\tilde{F}_{\alpha, N}$ is defined as

$$
\begin{aligned}
\tilde{F}_{\alpha, N}(\psi) & :=\left\langle\phi_{\mu}\left|H_{0}^{N}+\mu\right| \phi_{\mu}\right\rangle-\mu\|\psi\|_{L^{2}\left(\mathbb{R}^{3(N+1)}\right)}^{2}+\tilde{T}_{\alpha, \mu, N}(\vec{\xi}) \\
\tilde{T}_{\alpha, \mu, N}(\vec{\xi}) & :=\frac{2 m}{m+1} \alpha \sum_{i=1}^{N}\left\|\xi_{i}\right\|_{L^{2}\left(\mathbb{R}^{3 N}\right)}^{2}+\tilde{T}_{\text {dia }}^{\mu, N}(\vec{\xi})+\tilde{T}_{\text {off }}^{\mu, N}(\vec{\xi})
\end{aligned}
$$

where $\vec{\xi}=\left(\xi_{i}\right)_{i=1}^{N}$ and

$$
\begin{aligned}
& \tilde{T}_{\text {dia }}^{\mu, N}(\vec{\xi}):=\sum_{i=1}^{N} \int_{\mathbb{R}^{3 N}}\left|\hat{\xi}_{i}(\vec{k})\right|^{2} L_{\mu, N}(\vec{k}) \mathrm{d} \vec{k} \\
& \tilde{T}_{\text {off }}^{\mu, N}(\vec{\xi}):=-\sum_{\substack{i \neq j \\
1 \leq i, j \leq N}} \int_{\mathbb{R}^{3(N+1)}} \hat{\xi}_{i}^{*}\left(k_{0}+k_{i}, \hat{k}_{i}\right) \hat{\xi}_{j}\left(k_{0}+k_{j}, \hat{k}_{j}\right) G_{\mu}\left(k_{0}, \vec{k}\right) \mathrm{d} k_{0} \mathrm{~d} \vec{k} .
\end{aligned}
$$

Each $\xi_{i}$ in (3.2) corresponds to a function supported on the hyperplane $x_{0}=x_{i}$. The only overlap between hyperplanes for $i \neq j$ is on the set $x_{i}=x_{0}=x_{j}$ (of zero measure in $\mathbb{R}^{3 N}$ ), which implies that $\sum_{i=1}^{N} \hat{\xi}_{i}\left(k_{0}+k_{i}, \hat{k}_{i}\right)$ has a unique decomposition into $\left(\xi_{i}\right)_{i=1}^{N}$, and thus the splitting $\psi=\phi_{\mu}+\sum_{i=1}^{N} G_{\mu} \xi_{i}$ is unique. To stress the dependence on $\psi$, we will sometimes use the notation $\phi_{\mu}^{\psi}$ and $\xi_{i}^{\psi}$ below.

In the case that $\psi$ is antisymmetric in the last $N$ coordinates, the uniqueness of the decomposition $\psi=\phi_{\mu}+\sum_{i=1}^{N} G_{\mu} \xi_{i}$ shows that there exists a function $\xi \in H^{1 / 2}\left(\mathbb{R}^{3}\right) \otimes H_{\mathrm{as}}^{1 / 2}\left(\mathbb{R}^{3(N-1)}\right)$ such that $\xi_{i}=(-1)^{i+1} \xi$, and hence $\sum_{i=1}^{N} G_{\mu} \xi_{i}=G_{\mu} \xi$, which is defined in (1.5). Furthermore, we have

$$
\tilde{T}_{\text {dia }}^{\mu, N}(\vec{\xi})=N T_{\text {dia }}^{\mu, N}(\xi), \quad \tilde{T}_{\text {off }}^{\mu, N}(\vec{\xi})=N T_{\text {off }}^{\mu, N}(\xi)
$$

in this case, which shows that $\tilde{F}_{\alpha, N}(\psi)=F_{\alpha, N}(\psi)$ for $\psi$ antisymmetric in the last $N$ coordinates. In particular, $\tilde{F}_{\alpha, N}$ is an extension of $F_{\alpha, N}$, and for a lower bound it therefore suffices to work with $\tilde{F}_{\alpha, N}$.

In the following, it will be convenient to introduce the notation

$$
\tilde{\nabla}:=\left(\frac{1}{\sqrt{2 m}} \nabla_{0}, \frac{1}{\sqrt{2}} \nabla_{1}, \ldots, \frac{1}{\sqrt{2}} \nabla_{N}\right)
$$

as well as

$$
H_{\mu}:=H_{0}^{N}+\mu=-\tilde{\nabla}^{2}+\mu .
$$

\subsection{Localization of Wavefunctions}

An important ingredient in the proof of Theorem 2.1 will be to localize the particles. For this purpose, we will study in this subsection how the splitting $\psi=\phi_{\mu}^{\psi}+\sum_{i=1}^{N} G_{\mu} \xi_{i}^{\psi}$ is affected when multiplying $\psi$ by a smooth function.

Lemma 3.1. For $J \in \mathcal{C}^{\infty}\left(\mathbb{R}^{3(N+1)}\right)$ bounded and with bounded derivatives, we define $J \vec{\xi}=\left(J \xi_{i}\right)_{i=1}^{N}$ by

$$
\left(J \xi_{i}\right)\left(x_{i}, \hat{x}_{i}\right)=J\left(x_{i}, \vec{x}\right) \xi_{i}\left(x_{i}, \hat{x}_{i}\right) .
$$


Then, $\xi_{i} \mapsto\left[J, G_{\mu}\right] \xi_{i}:=J G_{\mu} \xi_{i}-G_{\mu} J \xi_{i}$ is a bounded map from $L^{2}\left(\mathbb{R}^{3 N}\right)$ to $H^{1}\left(\mathbb{R}^{3(N+1)}\right)$. In particular

$$
\xi_{i}^{J \psi}=J \xi_{i}^{\psi}
$$

and the regular part $\phi_{\mu}^{J \psi}$ of $J \psi$ is given by

$$
\phi_{\mu}^{J \psi}=J \phi_{\mu}^{\psi}+\sum_{i=1}^{N}\left[J, G_{\mu}\right] \xi_{i}^{\psi} .
$$

Remark. We clarify that $J$ acts on functions on $\mathbb{R}^{3(N+1)}$, and in particular on $\phi_{\mu}^{\psi}$ and $G_{\mu} \xi_{i}^{\psi}$, as a multiplication operator, whereas on functions in $L^{2}\left(\mathbb{R}^{3 N}\right)$ it acts as in (3.10), i.e., as multiplication by the function restricted to the relevant plane $\left\{x_{i}=x_{0}\right\}$. Hence, the commutator $\left[J, G_{\mu}\right]$ has no meaning here independently of its application on $\vec{\xi}$ and is only used as a convenient notation.

Proof. We first argue that $\left[J, G_{\mu}\right] \xi_{i}^{\psi} \in H^{1}\left(\mathbb{R}^{3(N+1)}\right)$ implies (3.11) and (3.12). We have

$$
J \psi-\sum_{i=1}^{N} G_{\mu} J \xi_{i}^{\psi}=J \phi_{\mu}^{\psi}+\sum_{i=1}^{N}\left[J, G_{\mu}\right] \xi_{i}^{\psi} .
$$

Since $J \phi_{\mu}^{\psi}$ and $\left[J, G_{\mu}\right] \xi_{i}^{\psi}$ are in $H^{1}\left(\mathbb{R}^{3(N+1)}\right)$, the uniqueness of the decomposition of $J \psi$ into regular and singular parts implies (3.11) and (3.12).

It remains to show that $\left[J, G_{\mu}\right] \xi_{i} \in H^{1}\left(\mathbb{R}^{3(N+1)}\right)$ for $\xi_{i} \in L^{2}\left(\mathbb{R}^{3 N}\right)$. In order to do so, we shall in fact show that

$$
\left[J, G_{\mu}\right] \xi_{i}=H_{\mu}^{-1}\left[H_{0}^{N}, J\right] G_{\mu} \xi_{i}=H_{\mu}^{-1}\left(-2 \tilde{\nabla} \cdot(\tilde{\nabla} J)-\left(\tilde{\nabla}^{2} J\right)\right) G_{\mu} \xi_{i},
$$

where we used the notation introduced in (3.8) and (3.9). From (3.14), the $H^{1}$ property readily follows, using that

$$
\begin{aligned}
\left\|G_{\mu} \xi_{i}\right\|_{L^{2}\left(\mathbb{R}^{3(N+1)}\right)}^{2}=\int_{\mathbb{R}^{3(N+1)}} & G_{\mu}\left(k_{0}, \vec{k}\right)^{2}\left|\hat{\xi}_{i}\left(k_{0}+k_{i}, \hat{k}_{i}\right)\right|^{2} \mathrm{~d} k_{0} \mathrm{~d} \vec{k} \\
& \lesssim\left(\frac{m}{m+1}\right)^{3 / 2} \mu^{-1 / 2}\left\|\xi_{i}\right\|_{L^{2}\left(\mathbb{R}^{3 N}\right)}^{2} .
\end{aligned}
$$

In the last step, we did an explicit integration over $\frac{1}{m+1} k_{0}-\frac{m}{m+1} k_{i}$, the variable canonically conjugate to $x_{0}-x_{i}$.

In order to show (3.14), we note that since $J$ is smooth, $H_{\mu}^{-1} J H_{\mu}$ is a bounded operator. In the sense of distributions, we have

$$
\left(H_{\mu} G_{\mu} \xi_{i}\right)\left(x_{0}, \vec{x}\right)=\xi_{i}\left(x_{i}, \hat{x}_{i}\right) \delta\left(x_{0}-x_{i}\right)
$$

and hence $H_{\mu}^{-1} J H_{\mu} G_{\mu} \xi_{i}=G_{\mu} J \xi_{i}$. In particular,

$$
\left[J, G_{\mu}\right] \xi_{i}=\left(J-H_{\mu}^{-1} J H_{\mu}\right) G_{\mu} \xi_{i}
$$

which indeed equals (3.14). This completes the proof of the lemma.

Corollary 3.1. Assume that $\psi \in D\left(\tilde{F}_{\alpha, N}\right)$ satisfies $\operatorname{supp} \psi \subseteq \Omega_{0} \times \cdots \times \Omega_{N}$, where $\Omega_{j} \subseteq \mathbb{R}^{3}$ for $0 \leq j \leq N$. Then,

$$
\operatorname{supp} \xi_{i}^{\psi} \subseteq\left(\Omega_{0} \cap \Omega_{i}\right) \times \Omega_{1} \times \cdots \times \Omega_{i-1} \times \Omega_{i+1} \times \cdots \times \Omega_{N} .
$$


Proof. Let $J \in \mathcal{C}^{\infty}\left(\mathbb{R}^{3(N+1)}\right)$ such that $J\left(x_{0}, \vec{x}\right)=1$ for $\left(x_{0}, \vec{x}\right) \in \Omega_{0} \times \cdots \times \Omega_{N}$. Using Lemma 3.1 we get that

$$
\xi_{i}^{\psi}\left(x_{i}, \hat{x}_{i}\right)=\xi_{i}^{J \psi}\left(x_{i}, \hat{x}_{i}\right)=J\left(x_{i}, \vec{x}\right) \xi_{i}^{\psi}\left(x_{i}, \hat{x}_{i}\right) .
$$

Since this holds for all $J$ with the above property, the claim follows.

\subsection{Alternative Representation of the Singular Part}

The following Lemma gives an alternative representation of the singular part of the quadratic form, defined in (3.4). It will turn out to be useful in the proof of the IMS formula in the next subsection.

Lemma 3.2. For $\vec{\xi}=\left(\xi_{i}\right)_{i=1}^{N}$ with $\xi_{i} \in H^{1 / 2}\left(\mathbb{R}^{3 N}\right)$, the function

$$
\mathcal{I}(\nu):=\left\|\sum_{i=1}^{N} G_{\nu} \xi_{i}\right\|_{L^{2}\left(\mathbb{R}^{3(N+1)}\right)}^{2}-\pi^{2}\left(\frac{2 m}{m+1}\right)^{3 / 2} \frac{1}{\sqrt{\nu}} \sum_{i=1}^{N}\left\|\xi_{i}\right\|_{L^{2}\left(\mathbb{R}^{3 N}\right)}^{2}
$$

is integrable on $[\mu, \infty)$ for any $\mu>0$, and we have

$$
\tilde{T}_{\alpha, \mu, N}(\vec{\xi})=\left(\frac{2 m}{m+1} \alpha+2 \pi^{2}\left(\frac{2 m}{m+1}\right)^{3 / 2} \sqrt{\mu}\right) \sum_{i=1}^{N}\left\|\xi_{i}\right\|_{L^{2}\left(\mathbb{R}^{3 N}\right)}^{2}-\int_{\mu}^{\infty} \mathrm{d} \nu \mathcal{I}(\nu) .
$$

Proof. For any $1 \leq i \leq N$, we have

$$
\begin{aligned}
\left\|G_{\nu} \xi_{i}\right\|_{L^{2}\left(\mathbb{R}^{3(N+1)}\right)}^{2} & =\int_{\mathbb{R}^{3(N+1)}} G_{\nu}\left(k_{0}, \vec{k}\right)^{2}\left|\hat{\xi}_{i}\left(k_{0}+k_{i}, \hat{k}_{i}\right)\right|^{2} \mathrm{~d} k_{0} \mathrm{~d} \vec{k} \\
& =\left(\frac{2 m}{m+1}\right)^{3 / 2} \int_{\mathbb{R}^{3 N}} \frac{\pi^{2}}{\sqrt{\frac{k_{i}^{2}}{2(1+m)}+\frac{1}{2} \hat{k}_{i}^{2}+\nu}}\left|\hat{\xi}_{i}\left(k_{i}, \hat{k}_{i}\right)\right|^{2} \mathrm{~d} k_{0} \mathrm{~d} \vec{k}
\end{aligned}
$$

In particular,

$$
\left\|G_{\nu} \xi_{i}\right\|_{L^{2}\left(\mathbb{R}^{3(N+1)}\right)}^{2}-\left(\frac{2 m}{m+1}\right)^{3 / 2} \frac{\pi^{2}}{\sqrt{\nu}}\left\|\xi_{i}\right\|_{L^{2}\left(\mathbb{R}^{3 N}\right)}^{2} \leq 0
$$

and we have

$$
\begin{gathered}
-\int_{\mu}^{\infty} \mathrm{d} \nu\left(\left\|G_{\nu} \xi_{i}\right\|_{L^{2}\left(\mathbb{R}^{3(N+1)}\right)}^{2}-\left(\frac{2 m}{m+1}\right)^{3 / 2} \frac{\pi^{2}}{\sqrt{\nu}}\left\|\xi_{i}\right\|_{L^{2}\left(\mathbb{R}^{3 N}\right)}^{2}\right) \\
=\int_{\mathbb{R}^{3 N}}\left|\hat{\xi}_{i}(\vec{k})\right|^{2} L_{\mu, N}(\vec{k}) \mathrm{d} \vec{k}-2 \pi^{2}\left(\frac{2 m}{m+1}\right)^{3 / 2} \sqrt{\mu}\left\|\xi_{i}\right\|_{L^{2}\left(\mathbb{R}^{3 N}\right)}^{2} .
\end{gathered}
$$

For the terms $i \neq j$, on the other hand, we have

$$
\begin{aligned}
& \int_{\mu}^{\infty} \mathrm{d} \nu\left\langle G_{\nu} \xi_{i} \mid G_{\nu} \xi_{j}\right\rangle \\
& \quad=\int_{\mu}^{\infty} \mathrm{d} \nu \int_{\mathbb{R}^{3(N+1)}} \hat{\xi}_{i}^{*}\left(k_{0}+k_{i}, \hat{k}_{i}\right) \hat{\xi}_{j}\left(k_{0}+k_{j}, \hat{k}_{j}\right) G_{\nu}\left(k_{0}, \vec{k}\right)^{2} \mathrm{~d} k_{0} \mathrm{~d} \vec{k}
\end{aligned}
$$




$$
=\int_{\mathbb{R}^{3(N+1)}} \hat{\xi}_{i}^{*}\left(k_{0}+k_{i}, \hat{k}_{i}\right) \hat{\xi}_{j}\left(k_{0}+k_{j}, \hat{k}_{j}\right) G_{\mu}\left(k_{0}, \vec{k}\right) \mathrm{d} k_{0} \mathrm{~d} \vec{k} .
$$

Here, the exchange of the order of integration is justified by Fubini's theorem, since the integrand in the first line on the right is absolutely integrable for $\xi_{i} \in H^{1 / 2}$. This completes the proof.

\subsection{IMS Formula}

In this subsection, we will prove the following Lemma.

Proposition 3.1. Given $M \geq 1$ and $\left(J_{i}\right)_{i=1}^{M}$ with $J_{i} \in \mathcal{C}^{\infty}\left(\mathbb{R}^{3(N+1)}\right)$ and $\sum_{i=1}^{M} J_{i}^{2}=1$, we have

$$
\tilde{F}_{\alpha, N}(\psi)=\sum_{i=1}^{M} \tilde{F}_{\alpha, N}\left(J_{i} \psi\right)-\sum_{i=1}^{M}\left\|\left(\tilde{\nabla} J_{i}\right) \psi\right\|^{2}
$$

for all $\psi \in D\left(\tilde{F}_{\alpha, N}\right)$.

Proof. By using the polarization identity, we can extend $\tilde{F}_{\alpha, N}$ to a sesquilinear form, denoted as $\tilde{F}_{\alpha, N}\left(\psi_{1}, \psi_{2}\right)$. It suffices to prove that

$$
\tilde{F}_{\alpha, N}\left(J^{2} \psi, \psi\right)+\tilde{F}_{\alpha, N}\left(\psi, J^{2} \psi\right)-2 \tilde{F}_{\alpha, N}(J \psi, J \psi)=-2\|(\tilde{\nabla} J) \psi\|^{2}
$$

for smooth functions $J$, since then

$$
\begin{array}{r}
\tilde{F}_{\alpha, N}(\psi)=\frac{1}{2} \sum_{i=1}^{M}\left(\tilde{F}_{\alpha, N}\left(J_{i}^{2} \psi, \psi\right)+\tilde{F}_{\alpha, N}\left(\psi, J_{i}^{2} \psi\right)\right) \\
\stackrel{(3.27)}{=} \sum_{i=1}^{M} \tilde{F}_{\alpha, N}\left(J_{i} \psi, J_{i} \psi\right)-\sum_{i=1}^{M}\left\|\left(\tilde{\nabla} J_{i}\right) \psi\right\|^{2} .
\end{array}
$$

Recall the definition $H_{\mu}=H_{0}^{N}+\mu$. The left side of (3.27) equals

$$
\begin{aligned}
& \left\langle\phi_{\mu}^{J^{2} \psi}\left|H_{\mu}\right| \phi_{\mu}^{\psi}\right\rangle+\left\langle\phi_{\mu}^{\psi}\left|H_{\mu}\right| \phi_{\mu}^{J^{2} \psi}\right\rangle-2\left\langle\phi_{\mu}^{J \psi}\left|H_{\mu}\right| \phi_{\mu}^{J \psi}\right\rangle \\
& \quad+\tilde{T}_{\alpha, \mu, N}\left(\vec{\xi}^{J^{2} \psi}, \vec{\xi}^{\psi}\right)+\tilde{T}_{\alpha, \mu, N}\left(\vec{\xi}^{\psi}, \vec{\xi}^{J^{2} \psi}\right)-2 \tilde{T}_{\alpha, \mu, N}\left(\vec{\xi}^{J \psi}, \vec{\xi}^{J \psi}\right)
\end{aligned}
$$

where we introduced the sesquilinear form $\tilde{T}_{\alpha, \mu, N}\left(\vec{\xi}_{1}, \vec{\xi}_{2}\right)$ corresponding to the quadratic form (3.4). We use Lemma 3.1 to identify the regular and singular parts of the various wavefunctions. For the quadratic form $\tilde{T}_{\alpha, \mu, N}$, we utilize representation (3.21), which together with (3.11) implies that

$$
\begin{aligned}
& \tilde{T}_{\alpha, \mu, N}\left(\vec{\xi}^{J^{2} \psi}, \vec{\xi}^{\psi}\right)+\tilde{T}_{\alpha, \mu, N}\left(\vec{\xi}^{\psi}, \vec{\xi}^{J^{2} \psi}\right)-2 \tilde{T}_{\alpha, \mu, N}\left(\vec{\xi}^{J \psi}, \vec{\xi}^{J \psi}\right) \\
& =\int_{\mu}^{\infty} \mathrm{d} \nu \sum_{i, j=1}^{N}\left(2\left\langle G_{\nu} J \xi_{i}^{\psi} \mid G_{\nu} J \xi_{j}^{\psi}\right\rangle-\left\langle G_{\nu} J^{2} \xi_{i}^{\psi} \mid G_{\nu} \xi_{j}^{\psi}\right\rangle-\left\langle G_{\nu} \xi_{i}^{\psi} \mid G_{\nu} J^{2} \xi_{j}^{\psi}\right\rangle\right) .
\end{aligned}
$$

Since $G_{\nu} J \xi_{i}^{\psi}=H_{\nu}^{-1} J H_{\nu} G_{\nu} \xi^{\psi}$, as shown in the proof of Lemma 3.1, we can rewrite the terms in the integrand as 


$$
\begin{aligned}
& 2\left\langle G_{\nu} J \xi_{i}^{\psi} \mid G_{\nu} J \xi_{j}^{\psi}\right\rangle-\left\langle G_{\nu} J^{2} \xi_{i}^{\psi} \mid G_{\nu} \xi_{j}^{\psi}\right\rangle-\left\langle G_{\nu} \xi_{i}^{\psi} \mid G_{\nu} J^{2} \xi_{j}^{\psi}\right\rangle \\
& \quad=\left\langle G_{\nu} \xi_{i}^{\psi}\left|2 H_{\nu} J H_{\nu}^{-2} J H_{\nu}-H_{\nu}^{-1} J^{2} H_{\nu}-H_{\nu} J^{2} H_{\nu}^{-1}\right| G_{\nu} \xi_{j}^{\psi}\right\rangle .
\end{aligned}
$$

Using that $(\partial / \partial \nu) G_{\nu} \xi_{i}^{\psi}=-H_{\nu}^{-1} G_{\nu} \xi_{i}^{\psi}$ as well as $\left[J,\left[H_{\nu}, J\right]\right]=2|\tilde{\nabla} J|^{2}$, one readily checks that this further equals

$$
(3.31)=-2 \frac{\partial}{\partial \nu}\left\langle\left. G_{\nu} \xi_{i}^{\psi}\left|\left[J, H_{\nu}\right] H_{\nu}^{-1}\left[H_{\nu}, J\right]-\right| \tilde{\nabla} J\right|^{2} \mid G_{\nu} \xi_{j}^{\psi}\right\rangle .
$$

The operator $A_{\nu}:=\left[J, H_{\nu}\right] H_{\nu}^{-1}\left[H_{\nu}, J\right]-|\tilde{\nabla} J|^{2}$ is bounded, uniformly in $\nu$ for $\nu \geq \mu>0$. Since $\left\|G_{\nu} \xi_{i}^{\psi}\right\|_{2} \rightarrow 0$ as $\nu \rightarrow \infty$, we have $\lim _{\nu \rightarrow \infty}\left\langle G_{\nu} \xi_{i}^{\psi}\left|A_{\nu}\right| G_{\nu} \xi_{j}^{\psi}\right\rangle$ $=0$. In particular, from (3.30)-(3.32) we conclude that

$$
\begin{aligned}
& \tilde{T}_{\alpha, \mu, N}\left(\vec{\xi}^{J^{2} \psi}, \vec{\xi}^{\psi}\right)+\tilde{T}_{\alpha, \mu, N}\left(\vec{\xi}^{\psi}, \vec{\xi}^{J^{2} \psi}\right)-2 \tilde{T}_{\alpha, \mu, N}\left(\vec{\xi}^{J \psi}, \vec{\xi}^{J \psi}\right) \\
& \quad=\sum_{i, j=1}^{N}\left(2\left\langle G_{\mu} \xi_{i}^{\psi}\left|\left[J, H_{\mu}\right] H_{\mu}^{-1}\left[H_{\mu}, J\right]\right| G_{\mu} \xi_{j}^{\psi}\right\rangle-2\left\langle G_{\mu} \xi_{i}^{\psi} \|\left.\tilde{\nabla} J\right|^{2} G_{\mu} \xi_{j}^{\psi}\right\rangle\right) .
\end{aligned}
$$

For the regular part, we use (3.12) to rewrite the first line in (3.29) as

$$
\begin{aligned}
& \left\langle\phi_{\mu}^{J^{2} \psi}\left|H_{\mu}\right| \phi_{\mu}^{\psi}\right\rangle+\left\langle\phi_{\mu}^{\psi}\left|H_{\mu}\right| \phi_{\mu}^{J^{2} \psi}\right\rangle-2\left\langle\phi_{\mu}^{J \psi}\left|H_{\mu}\right| \phi_{\mu}^{J \psi}\right\rangle \\
& =-2\left\langle\left.\phi_{\mu}^{\psi}|| \tilde{\nabla} J\right|^{2} \phi_{\mu}^{\psi}\right\rangle-2 \sum_{i, j=1}^{N}\left\langle\left[J, G_{\mu}\right] \xi_{i}^{\psi}\left|H_{\mu}\right|\left[J, G_{\mu}\right] \xi_{j}^{\psi}\right\rangle \\
& \quad-4 \operatorname{Re} \sum_{i=1}^{N}\left\langle\left[J, G_{\mu}\right] \xi_{i}^{\psi}\left|H_{\mu}\right| J \phi_{\mu}^{\psi}\right\rangle+2 \operatorname{Re} \sum_{i=1}^{N}\left\langle\left[J^{2}, G_{\mu}\right] \xi_{i}^{\psi}\left|H_{\mu}\right| \phi_{\mu}^{\psi}\right\rangle .
\end{aligned}
$$

The second term on the right side equals

$$
-2 \sum_{i, j=1}^{N}\left\langle G_{\mu} \xi_{i}^{\psi}\left|\left[J, H_{\mu}\right] H_{\mu}^{-1}\left[H_{\mu}, J\right]\right| G_{\mu} \xi_{j}^{\psi}\right\rangle
$$

as (3.14) shows. Also the last line in (3.34) can be evaluated with the aid of (3.14), with the result that

$$
\begin{aligned}
& -4 \operatorname{Re} \sum_{i=1}^{N}\left\langle\left[J, G_{\mu}\right] \xi_{i}^{\psi}\left|H_{\mu}\right| J \phi_{\mu}^{\psi}\right\rangle+2 \operatorname{Re} \sum_{i=1}^{N}\left\langle\left[J^{2}, G_{\mu}\right] \xi_{i}^{\psi}\left|H_{\mu}\right| \phi_{\mu}^{\psi}\right\rangle \\
& =-4 \operatorname{Re} \sum_{i=1}^{N}\left\langle\left. G_{\mu} \xi_{i}^{\psi}|| \tilde{\nabla} J\right|^{2} \phi_{\mu}^{\psi}\right\rangle .
\end{aligned}
$$

In combination, (3.33), (3.34) and (3.35) imply the desired identity (3.27). This completes the proof of the lemma. 


\section{A Rough Bound}

In this section, we give a rough lower bound on the ground-state energy of $F_{\alpha, N}$ when restricted to wavefunctions $\psi \in D\left(F_{\alpha, N}\right)$ that are supported in $\mathcal{C}_{\ell}^{N+1}$ with $\mathcal{C}_{\ell}=(0, \ell)^{3}$ for some $\ell>0$. This lower bound has the desired scaling in $N$ and $\ell$, i.e., it is proportional to $N^{5 / 3} \ell^{-2}$, but with a non-sharp prefactor. For its proof, we will first reformulate the problem using periodic boundary conditions, and then apply the methods previously introduced in [25] to show stability in infinite space.

The statement of the following theorem involves three positive constants $c_{T}, c_{L}$ and $c_{\Lambda}$, which are independent of $m, N, \ell$ and $\alpha$ and which will be defined later. In particular, $c_{T}$ is defined in Eq. (4.44), $c_{L}$ in Eq. (4.84) and $c_{\Lambda}$ in Lemma 4.7.

Theorem 4.1. Let $\psi \in D\left(F_{\alpha, N}\right)$ with $\|\psi\|=1$ and $\operatorname{supp} \psi \subset(0, \ell)^{3(N+1)}$ for some $\ell>0$. Given $m>0$ and $\kappa>0$ such that

$$
1-\kappa / c_{T}>\Lambda(m)
$$

let $N_{0}=N_{0}(m, \kappa)$ be defined as

$$
N_{0}(m, \kappa)=\left(\left(1-\kappa / c_{T}-\Lambda(m)\right) \frac{m\left(1-\kappa / c_{T}\right)^{2}}{c_{\Lambda}}\right)^{-9 / 2} .
$$

For $N>N_{0}$, we have

$$
F_{\alpha, N}(\psi) \geq \kappa N^{5 / 3} \ell^{-2}-\frac{1}{4 \pi^{4}} \frac{m+1}{2 m} \frac{\left[\alpha-c_{L} \ell^{-1}\right]_{-}^{2}}{\left(1-\kappa / c_{T}-\Lambda(m)\right)^{2}\left(1-\left(N_{0} / N\right)^{2 / 9}\right)^{2}} .
$$

We note that this result gives a lower bound only for particle numbers $N>N_{0}(m, \kappa)$. In the case that $N \leq N_{0}$, we can still use $(2.2)$, however.

The remainder of this section contains the proof of Theorem 4.1. An important role will be played by a reformulation using periodic boundary conditions. We will start by introducing the functional $\tilde{F}_{\alpha, N}^{\text {per }}$ which is defined for periodic functions. In Lemma 4.2 , we will show that it is in fact equivalent to the original quadratic form $\tilde{F}_{\alpha, N}$ when applied to wavefunctions with compact support in $\mathcal{C}_{\ell}^{N+1}$. Working with periodic boundary conditions comes with the inconvenience of having to work with sums, rather than with integrals, in momentum space. In particular, this makes the explicit form of the singular part of $\tilde{F}_{\alpha, N}^{\text {per }}$ rather complicated; we shall compare it with the singular part of $\tilde{F}_{\alpha, N}$ in Lemma 4.4 and bound the difference. It comes with the big advantage of allowing us to choose $\mu$ negative, however, which will be essential to show a positive lower bound to the energy. This latter property would also be true for Dirichlet boundary conditions; the simpler form of the Greens function of the Laplacian makes periodic ones more convenient, however. In particular, it allows to apply the method of [25], which gives positivity of the singular part of $F_{\alpha, N}^{\text {per }}$ for $\mu \geq-\kappa N^{5 / 3} \ell^{-2}$ for small enough $\kappa$, under a condition of the form $\tilde{\Lambda}(m, \kappa)<1$. In Lemmas 4.5-4.7, we investigate the difference between 
$\tilde{\Lambda}(m, \kappa)$ and $\Lambda(m)$. In the last subsection, we combine these results to prove Theorem 4.1.

\subsection{Periodic Boundary Conditions}

Given $\psi \in D\left(\tilde{F}_{\alpha, N}\right)$ such that $\operatorname{supp} \psi \subset \mathcal{C}_{\ell}^{N+1}$, we extend $\psi$ to a periodic function $\psi^{\text {per }}$, defined as

$$
\psi^{\text {per }}\left(x_{0}, \ldots, x_{N}\right)=\psi\left(\tau\left(x_{0}\right), \ldots, \tau\left(x_{N}\right)\right)
$$

with

$$
\tau(x)=\left(\tau\left(x^{1}\right), \tau\left(x^{2}\right), \tau\left(x^{3}\right)\right), \quad \tau(s):=\inf \left((s+\ell \mathbb{Z}) \cap \mathbb{R}_{+}\right) \quad \text { for } s \in \mathbb{R} .
$$

In the following, we shall rewrite the functional $\tilde{F}_{\alpha, N}(\psi)$ in terms of $\psi^{\text {per }}$. Compared to Dirichlet boundary conditions, periodic ones have the advantage that one can work easily in the associated momentum space, similar to the unconfined case. For this purpose, we define the lattice in momentum space as

$$
\mathbb{L}:=\frac{2 \pi}{\ell} \mathbb{Z}^{3} .
$$

The function $\psi^{\text {per }}$ is then determined by its Fourier coefficients $\hat{\psi}^{\text {per }}\left(k_{0}, \vec{k}\right)$, which can be viewed as a function $\mathbb{L}^{N+1} \rightarrow \mathbb{C}$.

Corollary 3.1 implies that $\operatorname{supp} \xi_{i} \subset \mathcal{C}_{\ell}^{N}$ for all $1 \leq i \leq N$. Hence, we can extend it in a similar way as $\psi$ to a periodic function $\xi^{\text {per }}$. In momentum space, we can write it as $\hat{\xi}^{\text {per }}: \mathbb{L}^{N} \rightarrow \mathbb{C}$. For periodic functions, $G_{\mu} \psi^{\text {per }}$ does not make sense anymore, but instead choosing $G_{\mu}^{\text {per }}$ as the Greens function of the Laplacian with periodic boundary conditions allows us to define $G_{\mu}^{\text {per }} \xi_{i}^{\text {per }}$ by the Fourier coefficients

$$
G_{\mu}^{\text {per } \xi_{i}^{\text {per }}}\left(k_{0}, \vec{k}\right)=G_{\mu}\left(k_{0}, \vec{k}\right) \hat{\xi}_{i}^{\text {per }}\left(k_{0}+k_{i}, \hat{k}_{i}\right) .
$$

In order to motivate the quadratic form introduced below, we note that the expression $L_{\mu, N}(\vec{k})$ in (1.10) originates from the limit

$$
L_{\mu, N}(\vec{k})=\lim _{R \rightarrow \infty}\left(\frac{8 \pi m R}{m+1}-\int_{|t| \leq R} \frac{1}{\tilde{H}_{0}\left(k_{1}, t, \hat{k}_{1}\right)+\mu} \mathrm{d} t\right)
$$

where $\tilde{H}_{0}$ is the non-interacting Hamiltonian in momentum space, expressed in terms of center-of-mass and relative coordinates for the pair $\left(k_{0}, k_{1}\right)$, i.e.,

$$
\begin{aligned}
\tilde{H}_{0}\left(s, t, \hat{k}_{1}\right): & =\hat{H}_{0}^{N}\left(\frac{m}{m+1} s+t, \frac{1}{m+1} s-t, \hat{k}_{1}\right) \\
& =\frac{1}{2(m+1)} s^{2}+\frac{1+m}{2 m} t^{2}+\frac{1}{2} \hat{k}_{1}^{2} .
\end{aligned}
$$

More generally, we have

Lemma 4.1. Let $\tau$ be a nonnegative function in $\mathcal{C}_{0}^{\infty}\left(\mathbb{R}^{3}\right)$ such that $\hat{\tau}(0)$ $=1, \hat{\tau}(p) \geq 0$ for all $p \in \mathbb{R}^{3}$ and

$$
\int_{\mathbb{R}^{3}}|t|^{-2} \tau(t) \mathrm{d} t=4 \pi
$$


Then,

$$
L_{\mu, N}(\vec{k})=\lim _{R \rightarrow \infty}\left[\frac{8 \pi m R}{m+1}-\int_{\mathbb{R}^{3}} \frac{1}{\tilde{H}_{0}\left(k_{1}, t, \hat{k}_{1}\right)+\mu} \hat{\tau}(t / R) \mathrm{d} t\right] .
$$

Proof. Let $\gamma=\frac{1}{2(m+1)} k_{1}^{2}+\frac{1}{2} \hat{k}_{1}^{2}+\mu$. Using (4.10) we observe that (4.11) is equivalent to

$$
\lim _{R \rightarrow \infty} \int_{\mathbb{R}^{3}} \frac{\gamma}{\left(\left(\frac{1+m}{2 m}\right) t^{2}+\gamma\right)\left(\frac{1+m}{2 m}\right) t^{2}} \hat{\tau}(t / R) \mathrm{d} t=L_{\mu, N}(\vec{k}) .
$$

Since $\hat{\tau}(0)=1$ and $\hat{\tau}(t) \leq 1$ for all other $t$, the result follows from dominated convergence.

When replacing integrals by sums, we have to keep in mind that a change of coordinates from $\left(k_{0}, k_{1}\right)$ to $s=k_{0}+k_{1}$ and $t=\frac{m}{m+1} k_{1}-\frac{1}{m+1} k_{0}$ changes the domain over which we have to take the sums. Whereas $s \in \mathbb{L}$, we have to sum for a fixed $s$ the variable $t$ over $\mathbb{L}^{s}:=\mathbb{L}+\frac{m s}{m+1}$. Let $\tau$ be chosen as in Lemma 4.1, and define

$$
L_{\mu, N}^{\mathrm{per}}(\vec{k}):=\lim _{R \rightarrow \infty}\left(\frac{8 \pi m R}{m+1}-\left(\frac{2 \pi}{\ell}\right)^{3} \sum_{p \in \mathbb{L}^{k_{1}}} \frac{1}{\tilde{H}_{0}\left(k_{1}, p, \hat{k}_{1}\right)+\mu} \hat{\tau}(p / R)\right) .
$$

We shall see below that this definition is actually independent of $\tau$. For us it will be important that $\tau$ has compact support; hence, a sharp cutoff in momentum space would not be suitable.

We shall now define $\tilde{F}_{\alpha, N}^{\text {per }}$ with domain

$$
\begin{gathered}
D\left(\tilde{F}_{\alpha, N}^{\text {per }}\right)=\left\{\psi^{\text {per }}=\phi_{\mu}^{\text {per }}+\sum_{i=1}^{N} G_{\mu}^{\text {per }} \xi_{i}^{\text {per }} \mid \phi_{\mu}^{\text {per }} \in H_{\text {per }}^{1}\left(\mathcal{C}_{\ell}^{N+1}\right),\right. \\
\left.\xi_{i}^{\text {per }} \in H_{\text {per }}^{1 / 2}\left(\mathcal{C}_{\ell}^{N}\right) \forall i, 1 \leq i \leq N\right\}
\end{gathered}
$$

where $H_{\text {per }}^{1}\left(\mathcal{C}_{\ell}^{N+1}\right)$ and $H_{\text {per }}^{1 / 2}\left(\mathcal{C}_{\ell}^{N}\right)$ denotes the spaces of functions defined by Fourier coefficients in $\ell^{2}\left(\mathbb{L},\left(1+p^{2}\right)\right)^{\otimes(N+1)}$ and $\ell^{2}\left(\mathbb{L},\left(1+p^{2}\right)^{1 / 2}\right)^{\otimes N}$, respectively. The quadratic form is given by

$$
\begin{gathered}
\tilde{F}_{\alpha, N}^{\text {per }}\left(\psi^{\text {per }}\right):=\int_{\mathcal{C}_{\ell}^{N+1}}\left(\left|\tilde{\nabla} \phi_{\mu}^{\text {per }}\right|^{2}+\mu\left|\phi_{\mu}^{\text {per }}\right|^{2}\right)-\mu\left\|\psi^{\text {per }}\right\|_{L^{2}\left(\mathcal{C}_{\ell}^{N+1}\right)}^{2}+\tilde{T}_{\alpha, \mu, N}^{\text {per }}\left(\vec{\xi}^{\text {per }}\right) \\
\tilde{T}_{\alpha, \mu, N}^{\text {per }}\left(\vec{\xi}^{\text {per }}\right):=\sum_{i=1}^{N} \frac{2 m}{m+1} \alpha\left\|\xi_{i}^{\text {per }}\right\|_{L^{2}\left(\mathcal{C}_{\ell}^{N}\right)}^{2}+\tilde{T}_{\text {dia }}^{\text {per }, \mu}, N\left(\vec{\xi}^{\text {per }}\right)+\tilde{T}_{\text {off }}^{\text {per }, \mu, N}\left(\vec{\xi}^{\text {per }}\right)
\end{gathered}
$$

where $\vec{\xi}^{\text {per }}=\left(\xi_{i}^{\text {per }}\right)_{i=1}^{N}, \tilde{\nabla}$ is defined in (3.8), and the singular parts of the quadratic form are given by

$$
\tilde{T}_{\text {dia }}^{\text {per }, \mu, N}\left(\vec{\xi}^{\text {per }}\right):=\sum_{i=1}^{N}\left(\frac{2 \pi}{\ell}\right)^{3 N} \sum_{\vec{k} \in \mathbb{L}^{N}}\left|\hat{\xi}_{i}^{\text {per }}(\vec{k})\right|^{2} L_{\mu, N}^{\text {per }}(\vec{k})
$$




$$
\begin{aligned}
& \tilde{T}_{\mathrm{off}}^{\text {per }, \mu, N}\left(\vec{\xi}^{\mathrm{per}}\right) \\
& :=-\sum_{\substack{i \neq j \\
1 \leq i, j \leq N}}\left(\frac{2 \pi}{\ell}\right)^{3(N+1)} \sum_{k_{0} \in \mathbb{L}, \vec{k} \in \mathbb{L}^{N}} \hat{\xi}_{j}^{\text {per* }}\left(k_{0}+k_{j}, \hat{k}_{j}\right) \hat{\xi}_{i}^{\text {per }}\left(k_{0}+k_{i}, \hat{k}_{i}\right) G_{\mu}\left(k_{0}, \vec{k}\right) .
\end{aligned}
$$

We also define $F_{\alpha, N}^{\text {per }}$ as the restriction of $\tilde{F}_{\alpha, N}^{\text {per }}$ to functions antisymmetric in the last $N$ coordinates. Further, we define $T_{\text {dia }}^{\text {per, } \mu, N}, T_{\text {off }}^{\text {per }, \mu, N}$ and $T_{\alpha, \mu, N}^{\text {per }}$ in the natural way similar to $T_{\text {dia }}^{\mu, N}, T_{\text {off }}^{\mu, N}$ and $T_{\alpha, \mu, N}$ originating from $\tilde{T}_{\text {dia }}^{\mu, N}, \tilde{T}_{\text {off }}^{\mu, N}$ and $\tilde{T}_{\alpha, \mu, N}$, respectively (compare with (1.7) and (3.7)).

Lemma 4.2. Let $\psi \in D\left(\tilde{F}_{\alpha, N}\right)$ be such that $\operatorname{supp} \psi \subset \mathcal{C}_{\ell}^{N+1}$. Then,

$$
\tilde{F}_{\alpha, N}^{\text {per }}\left(\psi^{\text {per }}\right)=\tilde{F}_{\alpha, N}(\psi) \text {. }
$$

Proof. Recall the splitting of $\psi$ into its regular and singular parts, and similarly for $\psi^{\text {per }}$ :

$$
\psi=\phi_{\mu}+\sum_{i} G_{\mu} \xi_{i}, \quad \psi^{\mathrm{per}}=\phi_{\mu}^{\mathrm{per}}+\sum_{i} G_{\mu}^{\mathrm{per}} \xi_{i}^{\mathrm{per}} .
$$

Recall also definition (3.9). In the sense of distributions, we can apply $H_{\mu}$ to $\phi_{\mu}$, and in particular $H_{\mu} \phi_{\mu} \in H^{-1}\left(\mathbb{R}^{3(N+1)}\right)$ as $\phi_{\mu} \in H^{1}\left(\mathbb{R}^{3(N+1)}\right)$. In this sense, we can write the regular part of $\tilde{F}_{\alpha, N}$ as $\left\langle\phi_{\mu} \mid H_{\mu} \phi_{\mu}\right\rangle$. Because supp $\psi \subset \mathcal{C}_{\ell}^{N+1}$ (and $\mathcal{C}_{\ell}$ is open by definition) we have $\varepsilon:=\operatorname{dist}\left(\operatorname{supp} \psi, \partial \mathcal{C}_{\ell}\right)>0$. Let $\chi$ be a smooth cutoff function such that $\chi(x)=1$ if $x \in \mathcal{C}_{\ell-\epsilon}:=[\varepsilon / 2, \ell-\varepsilon / 2]^{3}$ and $\chi(x)=0$ if $x \in \mathcal{C}_{\ell}^{c}$. As $\operatorname{supp}\left(H_{\mu} G_{\mu} \xi\right) \subseteq \mathcal{C}_{\ell-\epsilon}^{N+1}$ and $\operatorname{supp} \psi \subseteq \mathcal{C}_{\ell-\epsilon}^{N+1}$ also $\operatorname{supp}\left(H_{\mu} \phi_{\mu}\right) \subseteq \mathcal{C}_{\ell-\epsilon}^{N+1}$, and therefore

$$
\left\langle\phi_{\mu} \mid H_{\mu} \phi_{\mu}\right\rangle=\left\langle\chi \phi_{\mu} \mid H_{\mu} \phi_{\mu}\right\rangle \text {. }
$$

We use the identity $\chi \phi_{\mu}=\chi \phi_{\mu}^{\text {per }}+\chi \sum_{i=1}^{N} G_{\mu}^{\text {per }} \xi_{i}^{\text {per }}-\chi \sum_{i=1}^{N} G_{\mu} \xi_{i}$ as well as the fact that $H_{\mu} \phi_{\mu}=H_{\mu} \phi_{\mu}^{\text {per }}$ on $\mathcal{C}_{\ell-\epsilon}^{N+1}$ to obtain

$$
\begin{aligned}
(4.21) & =\left\langle\chi \phi_{\mu}^{\mathrm{per}} \mid H_{\mu} \phi_{\mu}^{\mathrm{per}}\right\rangle+\sum_{i=1}^{N}\left\langle\chi\left(G_{\mu}^{\mathrm{per}} \xi_{i}^{\mathrm{per}}-G_{\mu} \xi_{i}\right) \mid H_{\mu} \phi_{\mu}^{\mathrm{per}}\right\rangle \\
& =\int_{\mathcal{C}_{\ell}^{N+1}}\left(\left|\tilde{\nabla} \phi_{\mu}^{\mathrm{per}}\right|^{2}+\mu\left|\phi_{\mu}^{\mathrm{per}}\right|^{2}\right)+\sum_{i=1}^{N}\left\langle\chi\left(G_{\mu}^{\mathrm{per}} \xi_{i}^{\mathrm{per}}-G_{\mu} \xi_{i}\right) \mid H_{\mu} \phi_{\mu}^{\mathrm{per}}\right\rangle .
\end{aligned}
$$

Note that $H_{\mu} \chi\left(G_{\mu}^{\text {per }} \xi_{i}^{\text {per }}-G_{\mu} \xi_{i}\right)$ is supported on $\mathcal{C}_{\ell} \backslash \mathcal{C}_{\ell-\epsilon}$, and $\psi^{\text {per }}$ vanishes on this set. Hence,

$$
\sum_{i=1}^{N}\left\langle\chi\left(G_{\mu}^{\text {per }} \xi_{i}^{\text {per }}-G_{\mu} \xi_{i}\right) \mid H_{\mu} \phi_{\mu}^{\text {per }}\right\rangle=-\sum_{i, j=1}^{N}\left\langle G_{\mu}^{\text {per }} \xi_{i}^{\text {per }}-G_{\mu} \xi_{i} \mid \chi H_{\mu} G_{\mu}^{\text {per }} \xi_{j}^{\text {per }}\right\rangle .
$$

We claim that $(4.23)$ is equal to the difference $\tilde{T}_{\alpha, \mu, N}^{\text {per }}\left(\vec{\xi}^{\text {per }}\right)-\tilde{T}_{\alpha, \mu, N}(\vec{\xi})$. Let $\tau$ be given as in Lemma 4.1. We approximate the distribution 
$\left(\chi H_{\mu} G_{\mu}^{\text {per }} \xi_{j}^{\text {per }}\right)\left(x_{0}, \vec{x}\right)=\xi_{j}\left(x_{j}, \hat{x}_{j}\right) \delta\left(x_{j}-x_{0}\right)$ by the sequence of functions $\left(\xi_{j} \tau_{R}\right)\left(x_{0}, \vec{x}\right)=\xi_{j}\left(\left(m x_{j}+x_{0}\right) /(1+m), \hat{x}_{j}\right) \tau_{R}\left(x_{j}-x_{0}\right)$ with $\tau_{R}(x)=R^{3} \tau(R x)$. We assume that $R$ is large enough such that $\tau_{R}$ is supported in a ball of radius $\varepsilon / 2$, and hence $\xi_{j} \tau_{R}$ is supported in $\mathcal{C}_{\ell}^{N+1}$. Because $G_{\mu}^{\text {per }} \xi_{i}^{\text {per }}-G_{\mu} \xi_{i}$ is actually a smooth function, as $H_{\mu}\left(G_{\mu}^{\text {per }} \xi_{i}^{\text {per }}-G_{\mu} \xi_{i}\right)=0$ on $\mathcal{C}_{\ell}^{N+1}$, we conclude that (4.23) is equal to

$$
(4.23)=-\lim _{R \rightarrow \infty} \sum_{i, j=1}^{N}\left\langle G_{\mu}^{\text {per }} \xi_{i}^{\text {per }}-G_{\mu} \xi_{i} \mid \xi_{j} \tau_{R}\right\rangle .
$$

For the terms with $i \neq j$, we can use dominated convergence in momentum space to conclude that

$$
\lim _{R \rightarrow \infty} \sum_{i \neq j}\left\langle G_{\mu}^{\text {per }} \xi_{i}^{\text {per }}-G_{\mu} \xi_{i} \mid \xi_{j} \tau_{R}\right\rangle=\tilde{T}_{\text {off }}^{\mu, N}(\vec{\xi})-\tilde{T}_{\text {off }}^{\text {per }, \mu, N}\left(\vec{\xi}^{\text {per }}\right) .
$$

For the terms with $i=j$, we can further write

$$
\begin{aligned}
& \sum_{i=1}^{N}\left\langle G_{\mu}^{\mathrm{per}} \xi_{i}^{\mathrm{per}}-G_{\mu} \xi_{i} \mid \xi_{i} \tau_{R}\right\rangle \\
& \quad=\sum_{i=1}^{N}\left(\left\langle G_{\mu}^{\mathrm{per}} \xi_{i}^{\mathrm{per}} \mid \xi_{i} \tau_{R}\right\rangle-\frac{8 \pi m R}{m+1}\left\|\xi_{i}\right\|_{2}^{2}\right)-\sum_{i=1}^{N}\left(\left\langle G_{\mu} \xi_{i} \mid \xi_{i} \tau_{R}\right\rangle-\frac{8 \pi m R}{m+1}\left\|\xi_{i}\right\|_{2}^{2}\right) .
\end{aligned}
$$

Lemma 4.1 implies that the limit of the last two terms exists, is independent of the choice of $\tau$ and is equal to $\tilde{T}_{\text {dia }}^{\mu, N}(\vec{\xi})$. Because also (4.23) does not depend on $\tau$, we conclude that

$$
\lim _{R \rightarrow \infty} \sum_{i=1}^{N}\left(\left\langle G_{\mu}^{\mathrm{per}} \xi_{i}^{\mathrm{per}} \mid \xi_{i} \tau_{R}\right\rangle-\frac{8 \pi m R}{m+1}\left\|\xi_{i}\right\|_{2}^{2}\right)
$$

exists and is independent of $\tau$. Comparing with (4.13) and (4.17), we see that it actually equals $\tilde{T}_{\text {dia }}^{\text {per } \mu, N}\left(\vec{\xi}^{\text {per }}\right)$. Combining the above, we obtain

$$
\left\langle\phi_{\mu} \mid H_{\mu} \phi_{\mu}\right\rangle=\int_{\mathcal{C}_{\ell}^{N+1}}\left(\left|\tilde{\nabla} \phi_{\mu}^{\text {per }}\right|^{2}+\mu\left|\phi_{\mu}^{\text {per }}\right|^{2}\right)+\tilde{T}_{\alpha, \mu, N}^{\text {per }}\left(\vec{\xi}^{\text {per }}\right)-\tilde{T}_{\alpha, \mu, N}(\vec{\xi}) .
$$

This completes the proof of the lemma.

For fermions, described by wavefunctions $\psi^{\text {per }}$ that are antisymmetric in the last $N$ variables, the expression $G_{\mu}^{\text {per }} \xi^{\text {per }}$ in (4.7) is also well defined for negative $\mu$ as long as $\mu>-E_{N-1}^{\text {per }}$, where $E_{N-1}^{\text {per }}$ denotes the ground-state energy of the non-interacting Hamiltonian for $N-1$ fermions with periodic boundary conditions on $\partial \mathcal{C}_{\ell}$. (Note than $G_{\mu} \xi$, on the other hand, is only defined for $\mu>0$.) The following lemma shows that for such $\mu$ the quadratic form $F_{\alpha, N}^{\text {per }}$ is actually independent of $\mu$.

Lemma 4.3. For $\psi \in D\left(F_{\alpha, N}^{\mathrm{per}}\right)$ and $\mu>-E_{N-1}^{\mathrm{per}}$, the expression $F_{\alpha, N}^{\mathrm{per}}\left(\psi^{\mathrm{per}}\right)$ is well defined and independent of $\mu$. 
Proof. We first note that $G_{\mu}^{\text {per }} \xi^{\text {per }}$ is well defined for $\mu>-E_{N-1}^{\text {per }}$, because of the antisymmetry of $\xi^{\text {per }}$ in the last $N-1$ variables, which implies that $N-1$ of the variables $\left(k_{1}, \ldots, k_{N}\right)$ in $G_{\mu}\left(k_{0}, \vec{k}\right)$ in $(4.7)$ are actually different. For $\nu, \mu>-E_{N-1}^{\text {per }}$, we have

$$
\phi_{\mu}^{\text {per }}=\phi_{\nu}^{\text {per }}+G_{\nu}^{\text {per }} \xi^{\text {per }}-G_{\mu}^{\text {per }} \xi^{\text {per }} .
$$

Using the resolvent identity, we see that the regular part of the quadratic form satisfies

$$
\begin{aligned}
\int_{\mathcal{C}_{\ell}^{N+1}}\left(\left|\tilde{\nabla} \phi_{\mu}^{\text {per }}\right|^{2}+\mu\left|\phi_{\mu}^{\text {per }}\right|^{2}\right)= & \int_{\mathcal{C}_{\ell}^{N+1}}\left(\left|\tilde{\nabla} \phi_{\nu}^{\text {per }}\right|^{2}+\nu\left|\phi_{\nu}^{\text {per }}\right|^{2}\right)+(\mu-\nu)\left\|\phi_{\nu}^{\text {per }}\right\|^{2} \\
& +2(\mu-\nu) \operatorname{Re}\left\langle G_{\nu}^{\text {per }} \xi^{\text {per }} \mid \phi_{\nu}^{\text {per }}\right\rangle \\
& +(\mu-\nu)\left\langle G_{\nu}^{\text {per }} \xi^{\text {per }} \mid G_{\nu}^{\text {per }} \xi^{\text {per }}-G_{\mu}^{\text {per }} \xi^{\text {per }}\right\rangle .
\end{aligned}
$$

A straightforward computation using definitions (4.13)-(4.16) shows that

$$
T_{\alpha, \mu, N}^{\text {per }}\left(\xi^{\text {per }}\right)-T_{\alpha, \nu, N}^{\text {per }}\left(\xi^{\text {per }}\right)=(\mu-\nu)\left\langle G_{\nu}^{\text {per }} \xi^{\text {per }} \mid G_{\mu}^{\text {per }} \xi^{\text {per }}\right\rangle .
$$

Combining both statements yields the desired identity

$$
\begin{aligned}
& \int_{\mathcal{C}_{\ell}^{N+1}}\left(\left|\tilde{\nabla} \phi_{\mu}^{\text {per }}\right|^{2}+\mu\left|\phi_{\mu}^{\text {per }}\right|^{2}\right)-\mu\left\|\psi^{\text {per }}\right\|^{2}+T_{\alpha, \mu, N}^{\text {per }}\left(\xi^{\text {per }}\right) \\
& =\int_{\mathcal{C}_{\ell}^{N+1}}\left(\left|\tilde{\nabla} \phi_{\nu}^{\text {per }}\right|^{2}+\nu\left|\phi_{\nu}^{\text {per }}\right|^{2}\right)-\nu\left\|\psi^{\text {per }}\right\|^{2}+T_{\alpha, \nu, N}^{\text {per }}\left(\xi^{\text {per }}\right) .
\end{aligned}
$$

\subsection{Approximation by Integrals}

In the previous subsection, we have shown that the original and the periodic formulations of the energy functionals, $\tilde{F}_{\alpha, N}$ and $\tilde{F}_{\alpha, N}^{\text {per }}$, agree if applied to functions $\psi$ compactly supported in $\mathcal{C}_{\ell}^{N+1}$. One complication in the periodic form is that $L_{\mu, N}^{\mathrm{per}}$ is not given as explicitly as $L_{\mu, N}$. The following lemma gives a bound on the difference.

Lemma 4.4. Given $\mu$ and $\vec{q}$ such that

$$
Q_{\mu}^{2}:=\frac{1}{2} \sum_{i=2}^{N} q_{i}^{2}+\mu>0
$$

we have

$$
\left|L_{\mu, N}^{\mathrm{per}}\left(q_{1}, \hat{q}_{1}\right)-L_{\mu, N}\left(q_{1}, \hat{q}_{1}\right)\right| \leq c_{L}^{\prime} \frac{1}{Q_{\mu}^{2} \ell^{3}}
$$

where the constant $c_{L}^{\prime}$ is independent of $N, \vec{q}, m, \ell$ and $\mu$.

Proof. We recall the definitions of $L_{\mu, N}$ and $L_{\mu, N}^{\text {per }}$ for some arbitrary $\tau$ fulfilling the requirements of Lemma 4.1:

$$
L_{\mu, N}(\vec{q})=-\lim _{R \rightarrow \infty}\left(\int \frac{1}{\tilde{H}_{0}\left(q_{1}, s, \hat{q}_{1}\right)+\mu} \hat{\tau}(s / R) \mathrm{d} s-\frac{8 \pi m R}{m+1}\right)
$$




$$
L_{\mu, N}^{\mathrm{per}}(\vec{q})=-\lim _{R \rightarrow \infty}\left(\left(\frac{2 \pi}{\ell}\right)^{3} \sum_{s \in \mathbb{L}^{q_{1}}} \frac{1}{\tilde{H}_{0}\left(q_{1}, s, \hat{q}_{1}\right)+\mu} \hat{\tau}(s / R)-\frac{8 \pi m R}{m+1}\right)
$$

with $\tilde{H}_{0}$ defined in (4.9). For simplicity, we assume that $q_{1}$ is such that $\mathbb{L}^{q_{1}}=$ $\mathbb{L}$, but all other cases work analogously as a shift in momentum space only introduces a phase factor in configuration space, which vanishes when taking absolute values. In the following, we denote $f_{\infty}(s)=\left(\tilde{H}_{0}\left(q_{1}, s, \hat{q}_{1}\right)+\mu\right)^{-1}$ and $f_{R}(s)=f_{\infty}(s) \hat{\tau}(s / R)$ and suppress the dependence on $\vec{q}$ for simplicity.

We can express the difference between the Riemann sum and the integral using Poisson's summation formula

$$
\begin{aligned}
\left(\frac{2 \pi}{\ell}\right)^{3} \sum_{s \in \mathbb{L}} f_{R}(s)-\int_{\mathbb{R}^{3}} f_{R}(s) \mathrm{d} s & =\frac{(2 \pi)^{3}}{\ell^{3}} \sum_{s \in \mathbb{L}} f_{R}(s)-(2 \pi)^{3 / 2} \hat{f}_{R}(0) \\
& =(2 \pi)^{3 / 2} \sum_{\substack{z \in \ell \mathbb{Z}^{3} \\
z \neq 0}} \hat{f}_{R}(z) .
\end{aligned}
$$

For short we write $\gamma:=\frac{1}{2(1+m)} q_{1}^{2}+\frac{1}{2} \hat{q}_{1}^{2}+\mu$, which is bounded from below by $Q_{\mu}^{2}$ and hence is positive, by our assumption (4.33). The function $f_{\infty}$ and its Fourier transform are given by

$$
f_{\infty}(t)=\frac{1}{\frac{1+m}{2 m} t^{2}+\gamma}, \quad \hat{f}_{\infty}(z)=\sqrt{\frac{\pi}{2}} \frac{2 m}{1+m} \frac{e^{-\left(\frac{2 m}{m+1}\right)^{1 / 2} \sqrt{\gamma}|z|}}{|z|} .
$$

Moreover,

$$
\hat{f}_{R}(z)=(2 \pi)^{-3 / 2}\left(R^{3} \tau(R \cdot) * \hat{f}_{\infty}\right)(z) .
$$

We will show that $\hat{f}_{R}(s)$ is summable over $\ell \mathbb{Z}^{3} \backslash\{0\}$. In fact for $|z| \gtrsim \ell$,

$$
\begin{aligned}
(2 \pi)^{3 / 2}\left|\hat{f}_{R}(z)\right|= & \int_{\mathbb{R}^{3}} R^{3} \tau(R w) \hat{f}_{\infty}(z-w) \mathrm{d} w \\
\leq & \int_{|w|>|z| / 2} R^{3} \tau(R w) \hat{f}_{\infty}(z-w) \mathrm{d} w \\
& +\int_{|z-w|>|z| / 2} R^{3} \tau(R w) \hat{f}_{\infty}(z-w) \mathrm{d} w \\
\leq & \hat{f}_{\infty}(z / 2) \int R^{3} \tau(R w) \mathrm{d} w=\hat{f}_{\infty}(z / 2)
\end{aligned}
$$

where we assumed that $R$ is large enough such that $\tau(R w)=0$ for $|w|>|z| / 2$, and used that $\int \tau=1$, which was required by Lemma 4.1. As $\hat{f}_{\infty}$ is summable over $\ell \mathbb{Z}^{3} \backslash\{0\}$, we get by dominated convergence that

$$
\lim _{R \rightarrow \infty} \sum_{z \in \ell \mathbb{Z}^{3} \backslash\{0\}}\left|\hat{f}_{R}(z)\right|=\sum_{z \in \ell \mathbb{Z}^{3} \backslash\{0\}} \hat{f}_{\infty}(z) .
$$

We bound the sum over $\hat{f}_{\infty}(z)$ by

$$
\sum_{z \in \ell \mathbb{Z}^{3} \backslash\{0\}} \hat{f}_{\infty}(z)=\sum_{n \in \mathbb{Z}^{3} \backslash\{0\}} \sqrt{\frac{\pi}{2}} \frac{2 m}{1+m} \frac{e^{-\left(\frac{2 m}{m+1}\right)^{1 / 2} \sqrt{\gamma} \ell|n|}}{\ell|n|} \lesssim \frac{1}{\gamma \ell^{3}}
$$


using

$$
\sum_{n \in \mathbb{Z}^{3} \backslash\{0\}} e^{-\eta|n|} /|n| \lesssim \sum_{n \in \mathbb{N}} n e^{-\eta n}=\frac{e^{-\eta}}{\left(1-e^{-\eta}\right)^{2}} \leq \frac{1}{\eta^{2}}
$$

for $\eta=(2 m /(m+1))^{1 / 2} \sqrt{\gamma} \ell$. Combining (4.36), (4.40) and (4.41) and using that $\gamma \geq Q_{\mu}^{2}$, we conclude that

$$
\lim _{R \rightarrow \infty}\left|\frac{(2 \pi)^{3}}{\ell^{3}} \sum_{s \in \mathbb{L}} f_{R}(s)-\int_{\mathbb{R}^{3}} f_{R}(s) \mathrm{d} s\right| \leq \frac{c_{L}^{\prime}}{\gamma \ell^{3}} \leq \frac{c_{L}^{\prime}}{Q_{\mu}^{2} \ell^{3}}
$$

for some constant $c_{L}^{\prime}>0$. This completes the proof of the lemma.

\subsection{Bound on the Singular Parts}

The strategy for obtaining a lower bound on $F_{\alpha, N}^{\text {per }}$ is to find a $\mu$ such that $T_{\alpha, \mu, N}^{\text {per }} \geq 0$, in which case we obtain the lower bound $F_{\alpha, N}^{\text {per }}\left(\psi^{\text {per }}\right) \geq-\mu\left\|\psi^{\text {per }}\right\|^{2}$. Hence, we want to choose $\mu$ as negative as possible. We shall use the method of [25], which yields the desired positivity of $T_{\alpha, \mu, N}^{\mathrm{per}}$ (for large enough $m$ ) as long as $\mu \geq-\kappa N^{5 / 3} \ell^{-2}$ for $\kappa$ small enough. (More precisely, $-\mu$ will be equal to the right side of (4.3).)

If we define $Q^{2}=\frac{1}{2} \sum_{i=2}^{N} q_{i}^{2}$ for $N>2$, we observe that there exists a constant $c_{T}>0$ such that

$$
Q^{2} \geq c_{T} N^{5 / 3} \ell^{-2}
$$

if all $q_{i} \in \mathbb{L}$ are different. We are only interested in values of $\vec{q}=\left(q_{1}, \ldots, q_{N}\right)$ where $\xi^{\operatorname{per}}(\vec{q})$ is nonzero, which requires all the $q_{i}$ for $i \geq 2$ to be different since $\xi^{\text {per }}$ is antisymmetric in these variables. (We note that in comparison with [25] $Q^{2}$ is defined with an additional factor $1 / 2$ here.) From now on, we restrict $\mu$ to satisfy $\mu \geq-\kappa N^{5 / 3} \ell^{-2}$ for some $\kappa<c_{T}$. This implies that

$$
Q_{\mu}^{2}=Q^{2}+\mu \geq\left(1-\kappa / c_{T}\right) Q^{2} \geq\left(c_{T}-\kappa\right) N^{5 / 3} \ell^{-2} .
$$

In particular, Lemma 4.4 yields the bound

$$
T_{\text {dia }}^{\text {per }, \mu, N}\left(\xi^{\text {per }}\right) \geq\left(\frac{2 \pi}{\ell}\right)^{3 N} \sum_{\vec{q} \in \mathbb{L}^{N}} L_{\mu, N}(\vec{q})\left|\hat{\xi}^{\text {per }}(\vec{q})\right|^{2}-\frac{1}{N^{5 / 3} \ell} \frac{c_{L}^{\prime}}{c_{T}-\kappa}\left\|\xi^{\text {per }}\right\|_{2}^{2}
$$

on the diagonal term of the singular part of $F_{\alpha, N}^{\text {per }}$. Following the same steps as in [25], we can obtain the following lower bound for the off-diagonal term.

Proposition 4.1. Assume that $\mu \geq-\kappa N^{5 / 3} \ell^{-2}$ for some $\kappa<c_{T}$. Then for all $\xi^{\text {per }} \in H_{\mathrm{per}}^{1 / 2}\left(\mathcal{C}_{\ell}^{3}\right) \otimes H_{\mathrm{as}, \mathrm{per}}^{1 / 2}\left(\mathcal{C}_{\ell}^{3(N-1)}\right)$, we have

$$
T_{\text {off }}^{\text {per }, \mu, N}\left(\xi^{\text {per }}\right) \geq-\frac{\tilde{\Lambda}(m, \kappa)}{1-\kappa / c_{T}}\left(\frac{2 \pi}{\ell}\right)^{3 N} \sum_{\vec{q} \in \mathbb{L}^{N}} L_{\mu, N}(\vec{q})\left|\hat{\xi}^{\text {per }}(\vec{q})\right|^{2}
$$

where

$$
\tilde{\Lambda}(m, \kappa):=\inf _{\delta>0} \sup _{\substack{\tilde{s}, K \in \mathbb{R}^{3} \\ Q_{\mu}^{2}>\left(c_{T}-\kappa\right) N^{5 / 3} \ell^{-2}}}\left(\frac{2 \pi}{\ell}\right)^{3} \sum_{\tilde{t} \in \mathbb{L}+A K} \lambda_{\tilde{s}, Q_{\mu}, K, m, \delta}(\tilde{t})
$$


with $A:=1 /(2+m)$ and

$$
\begin{aligned}
& \lambda_{\tilde{s}, Q_{\mu}, K, m, \delta}(\tilde{t}) \\
&:=\frac{(\tilde{s}-A K)^{2}+2 Q_{\mu}^{2}+N \delta \ell^{-2}}{\pi^{2}(1+m)}\left(\frac{m(m+2)}{(m+1)^{2}} \tilde{s}^{2}+\frac{m}{m+1}\left(2 Q_{\mu}^{2}+A K^{2}\right)\right)^{-1 / 4} \\
& \times \frac{1}{(\tilde{t}-A K)^{2}+\delta \ell^{-2}}\left(\frac{m(m+2)}{(m+1)^{2}} \tilde{t}^{2}+\frac{m}{m+1}\left(2 Q_{\mu}^{2}+A K^{2}\right)\right)^{-1 / 4} \\
& \times \frac{|\tilde{s} \cdot \tilde{t}|}{\left[\tilde{s}^{2}+\tilde{t}^{2}+\frac{m}{1+m}\left(2 Q_{\mu}^{2}+A K^{2}\right)\right]^{2}-\left[\frac{2}{(1+m)} \tilde{s} \cdot \tilde{t}\right]^{2}} .
\end{aligned}
$$

Proof. The proof works in almost the exact same way as in [25]; hence, we will not spell out the details. The main difference is that we now have to write sums instead of integrals, and in particular this implies that we have to choose the weight function $h\left(s, \hat{q}_{1}\right)$ (see $[25$, Eq. (4.12)]) differently, namely as

$$
h\left(s, \hat{q}_{1}\right)=\left(s^{2}+\delta \ell^{-2}\right) \prod_{i=2}^{N}\left(q_{i}^{2}+\delta \ell^{-2}\right) .
$$

For comparison, $\delta=0$ was used in [25]. Following the proof in [25, Sect. 4], this choice gives a lower bound to the off-diagonal term of the form

$$
T_{\text {off }}^{\text {per }, \mu, N}\left(\xi^{\text {per }}\right) \geq-\tilde{\Lambda}_{\delta, \mu}(m)\left(\frac{2 \pi}{\ell}\right)^{3 N} \sum_{\vec{q} \in \mathbb{L}^{N}} L_{\mu, N}(\vec{q})\left|\hat{\xi}^{\text {per }}(\vec{q})\right|^{2}
$$

with a prefactor $\tilde{\Lambda}_{\delta, \mu}(m)$ equal to

$$
\begin{aligned}
\sup _{\tilde{s}, K \in \mathbb{R}^{3}, Q^{2}>c_{T} N^{5 / 3} \ell^{-2}}\left(\frac{2 \pi}{\ell}\right)^{3} \sum_{\tilde{t} \in \mathbb{L}+A K} \frac{(\tilde{s}-A K)^{2}+2 Q^{2}+N \delta \ell^{-2}}{\pi^{2}(1+m)} \\
\quad \times\left(\frac{m(m+2)}{(m+1)^{2}} \tilde{s}^{2}+\frac{m}{m+1}\left(2 Q^{2}+2 \mu+A K^{2}\right)\right)^{-1 / 4} \\
\quad \times \frac{1}{(\tilde{t}-A K)^{2}+\delta \ell^{-2}}\left(\frac{m(m+2)}{(m+1)^{2}} \tilde{t}^{2}+\frac{m}{m+1}\left(2 Q^{2}+2 \mu+A K^{2}\right)\right)^{-1 / 4} \\
\times \frac{|\tilde{s} \cdot \tilde{t}|}{\left[\tilde{s}^{2}+\tilde{t}^{2}+\frac{m}{1+m}\left(2 Q^{2}+2 \mu+A K^{2}\right)\right]^{2}-\left[\frac{2}{(1+m)} \tilde{s} \cdot \tilde{t}\right]^{2}} .
\end{aligned}
$$

Since (4.45) holds under our assumption on $\mu$, we can use $Q^{2} \leq Q_{\mu}^{2}(1-$ $\left.\kappa / c_{T}\right)^{-1}$ in the first numerator in $(4.52)$ to conclude that $\inf _{\delta>0} \tilde{\Lambda}_{\delta, \mu}(m) \leq$ $\left(1-\kappa / c_{T}\right)^{-1} \tilde{\Lambda}(m, \kappa)$, which yields the desired result.

\subsection{A Bound on $\tilde{\Lambda}(m, \kappa)$}

We will not evaluate $\tilde{\Lambda}(m, \kappa)$ directly, but we will compare it with $\Lambda(m)$, which is defined in [25, Eq. (2.8)] and which was already referred to in (2.2) above. 
The expression $\Lambda(m)$ can be written as

$$
\begin{aligned}
\Lambda(m):= & \sup _{\substack{\tilde{s}, K \in \mathbb{R}^{3} \\
Q_{\mu}^{2}>0}} \int_{\mathbb{R}^{3}} \lambda_{\tilde{s}, Q_{\mu}, K, m, 0}(\tilde{t}) \mathrm{d} \tilde{t} \\
= & \sup _{\substack{\tilde{s}, K \in \mathbb{R}^{3} \\
Q_{\mu}^{2}>\left(c_{T}-\kappa\right) N^{5 / 3} \ell^{-2}}} \int_{\mathbb{R}^{3}} \lambda_{\tilde{s}, Q_{\mu}, K, m, 0}(\tilde{t}) \mathrm{d} \tilde{t} .
\end{aligned}
$$

The additional constraint on $Q_{\mu}$ in the latter supremum has no effect because of the scaling properties of $\lambda_{\tilde{s}, Q_{\mu}, K, m, 0}$, specifically $\lambda_{\nu \tilde{s}, \nu Q_{\mu}, \nu K, m, 0}(\nu \tilde{t})$ $=\nu^{-3} \lambda_{\tilde{s}, Q_{\mu}, K, m, 0}(\tilde{t})$ for any $\nu>0$, which allows to fix one of the parameters when taking the supremum. Expression (4.48) differs from (4.53) by the nonzero value of $\delta$, as well as the sum instead of an integral. In the following lemmas, we will compare the two.

The next Lemma gives a pointwise bound on $\lambda_{\tilde{s}, Q_{\mu}, K, m, \delta}$. For its statement, it will be convenient to define $C_{\ell}(s)$ as the cube with side length $2 \pi / \ell$ centered at $s \in \mathbb{R}^{3}$, i.e.,

$$
C_{\ell}(s)=\left[-\frac{\pi}{\ell}, \frac{\pi}{\ell}\right]^{3}+s .
$$

Lemma 4.5. For $m \gtrsim 1$, we have for $t \in \mathbb{L} \backslash\{0\}$

$$
\lambda_{\tilde{s}, Q_{\mu}, K, m, \delta}(\tilde{t}) \lesssim \frac{1}{m} \frac{1}{t^{5 / 2}} \frac{s^{2}+2 Q_{\mu}^{2}+N \delta \ell^{-2}}{\left(s^{2}+2 Q_{\mu}^{2}\right)^{1 / 4}} \frac{1}{s^{2}+t^{2}+2 Q_{\mu}^{2}}
$$

where $\tilde{s}=s+A K$ and $\tilde{t}=t+A K$. Moreover,

$$
\ell^{-3} \sum_{\tilde{t} \in \mathbb{L}+A K} \max _{\tau \in C_{\ell}(\tilde{t})} \lambda_{\tilde{s}, Q_{\mu}, K, m, \delta}(\tau) \lesssim \frac{1}{m}\left(1+\frac{N \delta}{\ell^{2} Q_{\mu}^{2}}+\frac{1}{\delta \ell Q_{\mu}}+\frac{N}{\ell^{3} Q_{\mu}^{3}}\right) .
$$

Proof. For the pointwise bound (4.55), we will proceed similarly to [25, Sect. 6]. Using the Cauchy-Schwarz inequality, we have

$$
|\tilde{t} \cdot \tilde{s}| \leq \frac{1}{2}\left[\tilde{s}^{2}+\tilde{t}^{2}+\frac{m}{1+m}\left(2 Q_{\mu}^{2}+A K^{2}\right)\right]
$$

and also

$$
\begin{gathered}
{\left[\tilde{s}^{2}+\tilde{t}^{2}+\frac{m}{1+m}\left(2 Q_{\mu}^{2}+A K^{2}\right)\right]^{2}-\left[\frac{2}{(1+m)} \tilde{s} \cdot \tilde{t}\right]^{2}} \\
\geq \frac{m(m+2)}{(1+m)^{2}}\left[\tilde{s}^{2}+\tilde{t}^{2}+\frac{m}{1+m}\left(2 Q_{\mu}^{2}+A K^{2}\right)\right]^{2}
\end{gathered}
$$

By minimizing over $K$, we find that

$$
\tilde{s}^{2}+\tilde{t}^{2}+\frac{m}{1+m}\left(2 Q_{\mu}^{2}+A K^{2}\right) \geq \frac{m(2+m)}{2+4 m+m^{2}}\left[s^{2}+t^{2}+2 Q_{\mu}^{2}\right]
$$

and

$$
\frac{m(m+2)}{(m+1)^{2}} \tilde{s}^{2}+\frac{m}{m+1}\left(2 Q_{\mu}^{2}+A K^{2}\right) \geq \frac{m}{m+1}\left(s^{2}+2 Q_{\mu}^{2}\right)
$$


By combining these bounds we get for (4.49) the pointwise bound

$$
\begin{aligned}
\lambda_{\tilde{s}, Q_{\mu}, K, m, \delta}(\tilde{t}) \leq & \left(\frac{m+1}{m}\right)^{3 / 2} \frac{m^{2}+4 m+2}{2 \pi^{2} m(m+2)^{2}}\left(s^{2}+2 Q_{\mu}^{2}+N \delta \ell^{-2}\right) \\
& \times\left(s^{2}+2 Q_{\mu}^{2}\right)^{-1 / 4} \frac{1}{t^{2}+\delta \ell^{-2}}\left(t^{2}+2 Q_{\mu}^{2}\right)^{-1 / 4} \frac{1}{s^{2}+t^{2}+2 Q_{\mu}^{2}}
\end{aligned}
$$

from which (4.55) readily follows.

We denote the right side of (4.55) by $\lambda^{>}(t)=\lambda_{s, Q_{\mu}, K, m, \delta}^{>}(t)$, and we will write $\lambda(\tilde{t})=\lambda_{\tilde{s}, Q_{\mu}, K, m, \delta}(\tilde{t})$ in the following. That is, (4.55) reads $\lambda(\tilde{t}) \lesssim \lambda^{>}(t)$. First, we treat the term $\tilde{t}=A K$ in (4.56). Using (4.61), we can bound

$$
\ell^{-3} \lambda(\tilde{t}) \lesssim \frac{1}{m \delta \ell Q_{\mu}} \frac{s^{2}+2 Q_{\mu}^{2}+N \delta \ell^{-2}}{s^{2}+t^{2}+2 Q_{\mu}^{2}} \lesssim \frac{1}{m}\left(\frac{1}{\delta \ell Q_{\mu}}+\frac{N}{\ell^{3} Q_{\mu}^{3}}\right)
$$

for any $\tilde{t}$ and hence, in particular, for $\tilde{t} \in C_{\ell}(A K)$. For the case $0 \neq t \in \mathbb{L}$, we note that for $\tau_{1}, \tau_{2} \in C_{\ell}(t)$ the bound $\left|\tau_{1}\right| \leq \sqrt{11}\left|\tau_{2}\right|$ holds, and hence

$$
\lambda^{>}\left(\tau_{1}\right) \leq 11^{9 / 4} \lambda^{>}\left(\tau_{2}\right) .
$$

In particular, the maximal value of $\lambda^{>}$in $C_{\ell}(\tau)$ is dominated by the average value, and therefore

$$
\begin{aligned}
\ell^{-3} \sum_{\tilde{t} \in \mathbb{L}+A K} \max _{\tau \in C_{\ell}(\tilde{t})} \lambda(\tau) & \lesssim \ell^{-3} \sum_{\substack{t \in \mathbb{L} \\
t \neq 0}} \lambda^{>}(t)+\frac{1}{m}\left(\frac{1}{\delta \ell Q_{\mu}}+\frac{N}{\ell^{3} Q_{\mu}^{3}}\right) \\
& \lesssim \sum_{\substack{t \in \mathbb{L} \\
t \neq 0}} \int_{C_{\ell}(t)} \lambda^{>}(t) \mathrm{d} t+\frac{1}{m}\left(\frac{1}{\delta \ell Q_{\mu}}+\frac{N}{\ell^{3} Q_{\mu}^{3}}\right) \\
& \lesssim \int_{\mathbb{R}^{3}} \lambda^{>}(t) \mathrm{d} t+\frac{1}{m}\left(\frac{1}{\delta \ell Q_{\mu}}+\frac{N}{\ell^{3} Q_{\mu}^{3}}\right) .
\end{aligned}
$$

As a last step, we explicitly evaluate the integral, which results in the bound

$$
\int_{\mathbb{R}^{3}} \lambda^{>}(t) \mathrm{d} t \lesssim \frac{1}{m}\left(1+\frac{N \delta}{\ell^{2} Q_{\mu}^{2}}\right)
$$

This completes the proof of the lemma.

Lemma 4.6. For $m \gtrsim 1$, we have

$$
\begin{aligned}
& \left|\int_{\mathbb{R}^{3}} \lambda_{\tilde{s}, Q_{\mu}, K, m, \delta}(\tilde{t}) \mathrm{d} \tilde{t}-\left(\frac{2 \pi}{\ell}\right)^{3} \sum_{\tilde{t} \in \mathbb{L}+A K} \lambda_{\tilde{s}, Q_{\mu}, K, m, \delta}(\tilde{t})\right| \\
& \quad \lesssim \frac{1}{m}\left(\frac{1}{\ell Q_{\mu}}+\frac{1}{\delta^{1 / 2}}\right)\left(1+\frac{N \delta}{\ell^{2} Q_{\mu}^{2}}+\frac{1}{\delta \ell Q_{\mu}}+\frac{N}{\ell^{3} Q_{\mu}^{3}}\right) .
\end{aligned}
$$

Proof. As in the proof of the previous Lemma, we denote $\lambda(\tilde{t})=\lambda_{\tilde{s}, Q_{\mu}, K, m, \delta}(\tilde{t})$, and write it as 


$$
\begin{aligned}
\lambda(\tilde{t})= & c_{5}\left((\tilde{s}-A K)^{2}+2 Q_{\mu}^{2}+N \delta \ell^{-2}\right)\left(c_{1} \tilde{s}^{2}+c_{2} Q_{\mu}^{2}+c_{3} K^{2}\right)^{-1 / 4} \\
& \times \frac{1}{(\tilde{t}-A K)^{2}+\delta \ell^{-2}}\left(c_{1} \tilde{t}^{2}+c_{2} Q_{\mu}^{2}+c_{3} K^{2}\right)^{-1 / 4} \\
& \times \frac{|\tilde{s} \cdot \tilde{t}|}{\left(\tilde{s}^{2}+\tilde{t}^{2}+c_{2} Q_{\mu}^{2}+c_{3} K^{2}\right)^{2}-\left(c_{4} \tilde{s} \cdot \tilde{t}\right)^{2}}
\end{aligned}
$$

with appropriate coefficients $c_{1}, c_{2}, c_{3}, c_{4}, c_{5}$ depending on $m$. Its gradient equals

$$
\begin{aligned}
\nabla \lambda(\tilde{t})= & \underbrace{-2 \frac{\tilde{t}-A K}{(\tilde{t}-A K)^{2}+\delta \ell^{-2}} \lambda(\tilde{t})}_{\mathrm{I}}-\underbrace{\frac{1}{2} \frac{c_{1} \tilde{t}}{c_{1} \tilde{t}^{2}+c_{2} Q_{\mu}^{2}+c_{3} K^{2}} \lambda(\tilde{t})}_{\mathrm{II}} \\
& -\underbrace{\frac{4 \tilde{t}\left(\tilde{s}^{2}+\tilde{t}^{2}+c_{2} Q_{\mu}^{2}+c_{3} K^{2}\right)-2 c_{4}^{2} \tilde{s}(\tilde{s} \cdot \tilde{t})}{\left(\tilde{s}^{2}+\tilde{t}^{2}+c_{2} Q_{\mu}^{2}+c_{3} K^{2}\right)^{2}-\left(c_{4} \tilde{s} \cdot \tilde{t}\right)^{2}} \lambda(\tilde{t})}_{\text {III }}+\underbrace{\frac{\tilde{s}}{\tilde{t} \cdot \tilde{s}} \lambda(\tilde{t})}_{\mathrm{IV}} .
\end{aligned}
$$

We can quantify the difference between the Riemann sum and the integral by

$$
\left|\int_{\mathbb{R}^{3}} \lambda(\tilde{t}) \mathrm{d} \tilde{t}-\left(\frac{2 \pi}{\ell}\right)^{3} \sum_{\tilde{t} \in \mathbb{L}+A K} \lambda(\tilde{t})\right| \lesssim \ell^{-4} \sum_{\tilde{t} \in \mathbb{L}+A K} \max _{\tau \in C_{\ell}(\tilde{t})}|\nabla \lambda(\tau)| .
$$

With the aid of the triangle inequality, we can treat the terms I-IV separately.

We can bound I as

$$
|\mathrm{I}| \leq \frac{2}{\sqrt{(\tilde{t}-A K)^{2}+\delta \ell^{-2}}} \lambda(\tilde{t}) \leq \frac{2 \ell}{\delta^{1 / 2}} \lambda(\tilde{t}) .
$$

For the second term, we obtain

$$
|\mathrm{II}| \leq \frac{1}{2} \sqrt{\frac{c_{1}}{c_{2}}} \frac{1}{Q_{\mu}} \lambda(\tilde{t})=\frac{1}{2^{3 / 2}} \sqrt{\frac{m+2}{m+1}} \frac{1}{Q_{\mu}} \lambda(\tilde{t}) \lesssim \frac{1}{Q_{\mu}} \lambda(\tilde{t}) .
$$

For III, we use similar estimates as in Lemma 4.5 to get

$$
|\mathrm{III}| \lesssim \frac{|\tilde{t}|+|\tilde{s}|}{\tilde{s}^{2}+\tilde{t}^{2}+c_{2} Q_{\mu}^{2}+c_{3} K^{2}} \lambda(\tilde{t}) \lesssim \frac{1}{Q_{\mu}} \lambda(\tilde{t}) .
$$

Finally, for IV we have to proceed slightly differently. If we use

$$
|\tilde{s}| \leq \frac{1}{2 \sqrt{c}_{2} Q_{\mu}}\left(\tilde{s}^{2}+\tilde{t}^{2}+c_{2} Q_{\mu}^{2}+c_{3} K^{2}\right)
$$

instead of (4.57), we see that we can bound $|\mathrm{III}|$ from above by $Q_{\mu}^{-1}$ times the right side of (4.61). Using Lemma 4.5, we conclude that

$$
\begin{aligned}
(4.69) & \leq \ell^{-4} \sum_{\tilde{t} \in \mathbb{L}+A K} \max _{\tau \in C_{\ell}(\tilde{t})}(|\mathrm{I}|+|\mathrm{II}|+|\mathrm{III}|+|\mathrm{IV}|) \\
& \lesssim \frac{1}{m}\left(\frac{1}{\ell Q_{\mu}}+\frac{1}{\delta^{1 / 2}}\right)\left(1+\frac{N \delta}{\ell^{2} Q_{\mu}^{2}}+\frac{1}{\delta \ell Q_{\mu}}+\frac{N}{\ell^{3} Q_{\mu}^{3}}\right) .
\end{aligned}
$$


Here, we have used that bound (4.56) holds also with $\lambda_{\tilde{s}, Q_{\mu}, K, m, \delta}$ replaced by the right side of (4.61), as shown in the proof of Lemma 4.5. This completes the proof.

Lemma 4.7. There exists a $c_{\Lambda}>0$ such that

$$
\tilde{\Lambda}(m, \kappa) \leq \Lambda(m)+\frac{1}{m} \frac{c_{\Lambda}}{\left(1-\kappa / c_{T}\right)^{2}} N^{-2 / 9}
$$

whenever $\kappa<c_{T}$ and $\Lambda(m) \leq 1$, where $c_{T}$ is defined in (4.44).

Proof. We first note that $\Lambda(m) \leq 1$ implies $m \gtrsim 1$. Moreover, from definition (4.49) we have

$$
\lambda_{\tilde{s}, Q_{\mu}, K, m, \delta}(\tilde{t}) \leq\left(1+\frac{N \delta}{2 \ell^{2} Q_{\mu}^{2}}\right) \lambda_{\tilde{s}, Q_{\mu}, K, m, 0}(\tilde{t}) .
$$

Combining this with Lemma 4.6 and taking the supremum over $\tilde{s}, K$ and $Q_{\mu}^{2} \geq\left(c_{T}-\kappa\right) N^{5 / 3} \ell^{-2}$, we obtain

$$
\begin{aligned}
& \tilde{\Lambda}(m, \kappa)-\Lambda(m) \\
& \lesssim \frac{1}{m} \inf _{\delta>0} \sup _{Q_{\mu}^{2} \geq\left(c_{T}-\kappa\right) N^{5 / 3} \ell^{-2}}\left[\frac{N \delta}{\ell^{2} Q_{\mu}^{2}}+\left(\frac{1}{\ell Q_{\mu}}+\frac{1}{\delta^{1 / 2}}\right)\left(1+\frac{N \delta}{\ell^{2} Q_{\mu}^{2}}+\frac{1}{\delta \ell Q_{\mu}}+\frac{N}{\ell^{3} Q_{\mu}^{3}}\right)\right]
\end{aligned}
$$

where we also used that $\Lambda(m) \lesssim m^{-1}$ for $m \gtrsim 1$. The supremum over $Q_{\mu}$ is clearly achieved for $Q_{\mu}^{2}=\left(c_{T}-\kappa\right) N^{5 / 3} \ell^{-2}$. For an upper bound, we shall choose $\delta \sim N^{4 / 9}$, which yields the desired bound

$$
\tilde{\Lambda}(m, \kappa)-\Lambda(m) \lesssim \frac{1}{m}\left(c_{T}-\kappa\right)^{-2} N^{-2 / 9} .
$$

\subsection{Proof of Theorem 4.1}

Using Proposition 4.1, Eq. (4.46) and Lemma 4.7, we get the lower bound

$$
\begin{aligned}
N^{-1} T_{\alpha, \mu, N}^{\text {per }}\left(\xi^{\text {per }}\right) \geq & \left(\frac{2 m \alpha}{m+1}-\frac{1}{N^{5 / 3} \ell} \frac{c_{L}^{\prime}}{c_{T}-\kappa}\right)\left\|\xi^{\text {per }}\right\|^{2} \\
& +\frac{1}{1-\kappa / c_{T}}\left(1-\kappa / c_{T}-\Lambda(m)-\frac{c_{\Lambda} N^{-2 / 9}}{m\left(1-\kappa / c_{T}\right)^{2}}\right) \\
& \times\left(\frac{2 \pi}{\ell}\right)^{3 N} \sum_{\vec{q} \in \mathbb{L}^{N}} L_{\mu, N}(\vec{q})\left|\xi^{\text {per }}(\vec{q})\right|^{2}
\end{aligned}
$$

for any $0<\kappa<c_{T}$ and $\mu \geq-\kappa N^{5 / 3} / \ell^{2}$. Note that the coefficient in front of the last sum is positive for all $N>N_{0}(\kappa, m)$, defined in (4.2). If $\alpha$ is large enough such that also the first term on the right side of (4.79) is nonnegative, we conclude that $T_{\alpha, \mu, N}^{\text {per }}\left(\xi^{\text {per }}\right) \geq 0$.

In case $2 m \alpha<(m+1) c_{L}^{\prime}\left(c_{T}-\kappa\right)^{-1} N^{-5 / 3} \ell^{-1}$, on the other hand, we need to dominate the first term on the right side of (4.79) by the second. We use (4.44) to obtain the lower bound 


$$
L_{\mu, N}(\vec{q}) \geq 2 \pi^{2}\left(\frac{2 m}{m+1}\right)^{3 / 2} Q_{\mu} \geq 2 \pi^{2}\left(\frac{2 m}{m+1}\right)^{3 / 2} \sqrt{\mu+\kappa N^{5 / 3} \ell^{-2}} .
$$

In particular, if we choose

$$
\begin{aligned}
\mu= & -\kappa N^{5 / 3} \ell^{-2} \\
& +\frac{1}{4 \pi^{4}} \frac{m+1}{2 m} \frac{\left(1-\kappa / c_{T}\right)^{2}\left[\alpha-(2 m)^{-1}(m+1) c_{L}^{\prime}\left(c_{T}-\kappa\right)^{-1} N^{-5 / 3} \ell^{-1}\right]_{-}^{2}}{\left(1-\kappa / c_{T}-\Lambda(m)-c_{\Lambda} m^{-1}\left(1-\kappa / c_{T}\right)^{-2} N^{-2 / 9}\right)^{2}}
\end{aligned}
$$

we again conclude that $T_{\alpha, \mu, N}^{\text {per }}\left(\xi^{\text {per }}\right) \geq 0$.

Note that for our choice of $\mu$, satisfying in particular $\mu \geq-c_{T} N^{5 / 3} \ell^{-2}$, we have

$$
\int_{\mathcal{C}_{\ell}^{N+1}}\left(\left|\tilde{\nabla} \phi_{\mu}^{\text {per }}\right|^{2}+\mu\left|\phi_{\mu}^{\text {per }}\right|^{2}\right) \geq 0
$$

for all $\phi_{\mu}^{\text {per }} \in H_{\text {per }}^{1}\left(\mathcal{C}_{\ell}^{N+1}\right)$ that are antisymmetric in the last $N$ variables. Hence, the positivity of $T_{\alpha, \mu, N}^{\text {per }}\left(\xi^{\text {per }}\right)$ implies that $F_{\alpha, N}^{\text {per }}\left(\psi^{\text {per }}\right) \geq-\mu\left\|\psi^{\text {per }}\right\|^{2}$. In combination with Lemmas 4.2 and 4.3 , this completes the proof of Theorem 4.1. To simplify its statement, we have additionally used that

$$
\begin{array}{r}
\left(1-\kappa / c_{T}\right)^{2}\left[\alpha-(2 m)^{-1}(m+1) c_{L}^{\prime}\left(c_{T}-\kappa\right)^{-1} N^{-5 / 3} \ell^{-1}\right]_{-}^{2} \\
\leq\left[\alpha-(2 m)^{-1}(m+1) c_{L}^{\prime} c_{T}^{-1} \ell^{-1}\right]_{-}^{2}
\end{array}
$$

for $N \geq 1$, and defined

$$
c_{L}:=\frac{m^{* *}+1}{2 m^{* *}} \frac{c_{L}^{\prime}}{c_{T}}
$$

in Eq. (4.3), where $m^{* *} \approx 0.36$ is chosen such that $m \geq m^{* *}$ for $\Lambda(m) \leq 1$.

\section{Proof of Theorem 2.1}

In this section, we will give the proof of our main result, Theorem 2.1.

Let $\mathcal{C}_{L}=(0, L)^{3}$ and $\overline{\mathcal{C}}_{L}=\bigcup_{i}^{M} \overline{\mathcal{C}}_{i}$ a disjoint decomposition into cubes $\mathcal{C}_{i}=(0, \ell)^{3}+z_{i}$ with $z_{i} \in \mathbb{R}^{3}$. We will choose $\ell$ such that $L / \ell \in \mathbb{N}$ in which case $M=(L / \ell)^{3}$. Let $1 / 4>\varepsilon>0$ and let $\eta \in \mathcal{C}_{0}^{\infty}\left(B_{\varepsilon}(0)\right)$ be nonnegative, with $\eta(0)>0$, where we denote by $B_{\varepsilon}(0)$ the centered ball of radius $\varepsilon$. In the following, we will assume that $\varepsilon$ is a fixed constant independent of all parameters (for example $\varepsilon=1 / 8$ works). For $x \in \mathcal{C}_{L}$, define

$$
J_{i}(x)=\left(\frac{\int_{\mathcal{C}_{i}} \eta\left(\ell^{-1}(x-y)\right) \mathrm{d} y}{\int_{\mathcal{C}_{L}} \eta\left(\ell^{-1}(x-y)\right) \mathrm{d} y}\right)^{1 / 2}
$$

Note that since $\eta$ is assumed to be strictly positive in a small ball around the origin, the denominator in (5.1) is strictly positive for $x \in \overline{\mathcal{C}}_{L}$, hence (5.1) is well defined. We have supp $J_{i} \subseteq \mathcal{C}_{i}+B_{\ell \varepsilon}(0)$ and $J_{i}(x)=1$ for $x \in \ell(\varepsilon, 1-\varepsilon)^{3}+z_{i}$. Moreover, $\sum_{i=1}^{M} J_{i}^{2}(x)=1$ for $x \in \mathcal{C}_{L}$ by construction. The derivative of $J_{i}$ 
can be bounded uniformly in $i$ and $M$ by a constant $c_{\eta}$ depending only on $\eta$ (and hence $\varepsilon$ ) as

$$
\left|\nabla J_{i}\right|^{2} \leq \frac{c_{\eta}}{\ell^{2}}
$$

Let $\psi \in D\left(F_{\alpha, N}\right)$ be such that $\operatorname{supp} \psi \subset \mathcal{C}_{L}^{N+1}$ and $\|\psi\|_{2}=1$. We use the IMS formula, Proposition 3.1, for the quadratic form $F_{\alpha, N}$ to localize the impurity particle (with coordinate $x_{0}$ ). With $J_{i} \psi$ denoting the function $\left(J_{i} \psi\right)\left(x_{0}, \vec{x}\right)=J_{i}\left(x_{0}\right) \psi\left(x_{0}, \vec{x}\right)$ we obtain

$$
F_{\alpha, N}(\psi)=\sum_{i=1}^{M} F_{\alpha, N}\left(J_{i} \psi\right)-\frac{1}{2 m} \sum_{i=1}^{M} \int\left|\nabla J_{i}\left(x_{0}\right)\right|^{2}\left|\psi\left(x_{0}, \vec{x}\right)\right|^{2} \mathrm{~d} x_{0} \mathrm{~d} \vec{x} .
$$

We note that the last term is bounded by

$$
\sum_{i=1}^{M} \int\left|\nabla J_{i}\left(x_{0}\right)\right|^{2}\left|\psi\left(x_{0}, \vec{x}\right)\right|^{2} \leq \frac{c_{\eta}}{\ell^{2}} \sum_{i=1}^{M} \int_{\partial J_{i}}\left|\psi\left(x_{0}, \vec{x}\right)\right|^{2} \mathrm{~d} x_{0} \mathrm{~d} \vec{x} \leq \frac{8 c_{\eta}}{\ell^{2}}
$$

since $\varepsilon<1 / 2$, where $\partial J_{i}=\operatorname{supp}\left|\nabla J_{i}\right|$. Recall the definition of the mean density, $\bar{\rho}=N L^{-3}$. We will choose $\ell \sim \bar{\rho}^{-1 / 3}$ which means that (5.4) is of the order $\bar{\rho}^{2 / 3}$.

In the next step, we want to localize the other particles, to be able to distinguish whether they are close to the impurity or far from it. Because we violate the antisymmetry constraint by doing so, we will work with the extended quadratic form $\tilde{F}_{\alpha, N}$ defined in (3.4). Let $V \in \mathcal{C}_{0}^{\infty}\left(\mathbb{R}^{3}\right)$ satisfy 0 $\leq V \leq 1$, with $\operatorname{supp} V \subseteq[-2 \varepsilon, 1+2 \varepsilon]^{3}$ and $V(x)=1$ for $x \in[-\varepsilon, 1+\varepsilon]^{3}$. We define $V_{i}(x)=V\left(\left(x-z_{i}\right) / \ell\right)$ and $\tilde{V}_{i}(x):=\sqrt{1-V_{i}(x)^{2}}$. Figure 1 visualizes this setup.

We localize all the remaining particles using the IMS formula in Proposition 3.1, with the localization functions

$$
\left(x_{1}, \ldots, x_{N}\right) \mapsto \prod_{j \in A} V_{i}\left(x_{j}\right) \prod_{k \in A^{c}} \tilde{V}_{i}\left(x_{k}\right)
$$

for $A \subseteq\{1, \ldots, N\}$, where $A^{c}=\{1, \ldots, N\} \backslash A$. For short we define

$$
\varphi_{i, A}\left(x_{0}, \vec{x}\right):=J_{i}\left(x_{0}\right) \prod_{k \in A} V_{i}\left(x_{k}\right) \prod_{j \in A^{c}} \tilde{V}_{i}\left(x_{j}\right) \psi\left(x_{0}, \vec{x}\right) .
$$

A straightforward calculation using Proposition 3.1 and the fact that $V_{i}^{2}+\tilde{V}_{i}^{2}$ $=1$ shows that

$$
\begin{aligned}
& F_{\alpha, N}\left(J_{i} \psi\right)=\sum_{A \subseteq\{1, \ldots, N\}}\left(\tilde{F}_{\alpha, N}\left(\varphi_{i, A}\right)\right. \\
& \left.-\frac{1}{2} \sum_{j=1}^{N} \int\left(\left|\nabla V_{i}\left(x_{j}\right)\right|^{2}+\left|\nabla \tilde{V}_{i}\left(x_{j}\right)\right|^{2}\right)\left|\varphi_{i, A}\left(x_{0}, \vec{x}\right)\right|^{2} \mathrm{~d} x_{0} \mathrm{~d} \vec{x}\right) .
\end{aligned}
$$

Here, it is necessary to introduce the extended quadratic form $\tilde{F}_{\alpha, N}$ since the functions $\varphi_{i, A}$ are not antisymmetric in all $N$ variables $\left(x_{1}, \ldots, x_{N}\right)$. They are 


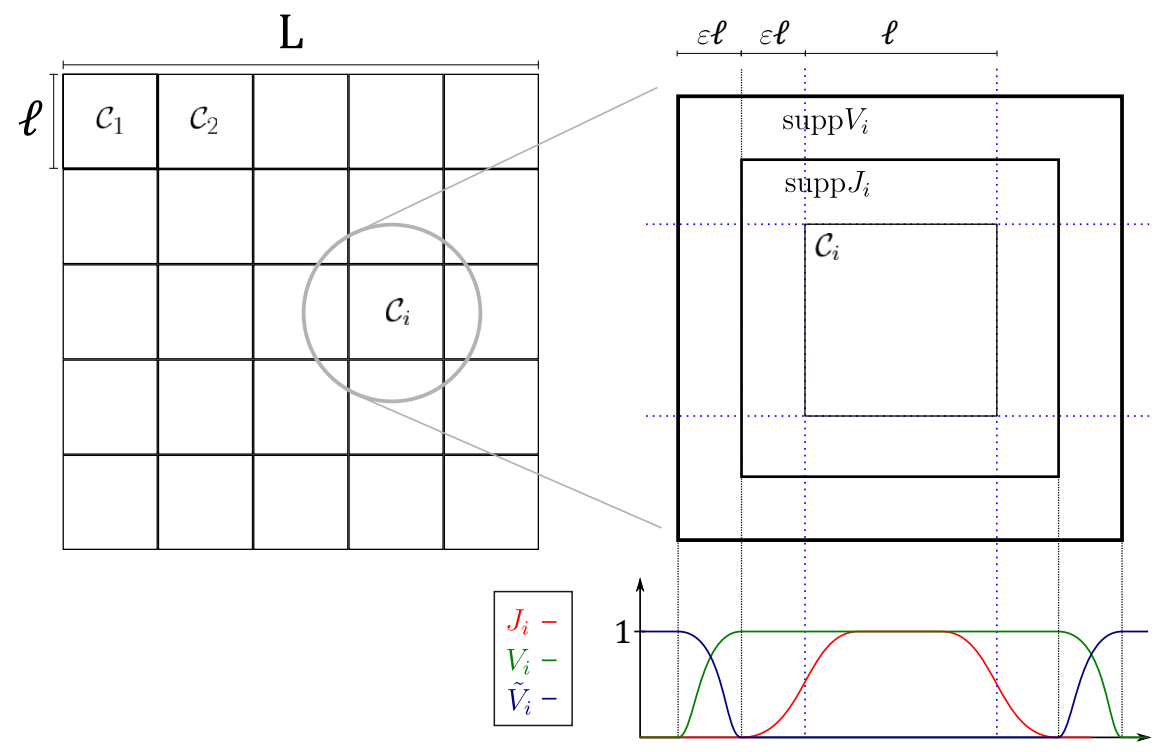

Figure 1. A sketch of the setup, the partitions $J_{i}, V_{i}, \tilde{V}_{i}$ and their boxes of support

still separately antisymmetric in the coordinates in $A$ and in the ones in $A^{c}$, however.

In the next lemma, we will show that the energy $\tilde{F}_{\alpha, N}\left(\varphi_{i, A}\right)$ splits up into a non-interacting energy for the particles in $A^{c}$ that are localized away from the impurity, and in a point-interacting quadratic form for particles in $A$.

Lemma 5.1. We define the functions $\varphi_{i, A}^{\vec{p}_{A^{c}}} \in L^{2}\left(\mathbb{R}^{3(|A|+1)}\right)$ and $\varphi_{i, A}^{p_{0}, \vec{p}_{A}}$ $\in L^{2}\left(\mathbb{R}^{3\left|A^{c}\right|}\right)$ via their Fourier transforms as

$$
\hat{\varphi}_{i, A}^{\vec{p}_{A^{c}}}\left(p_{0}, \vec{p}_{A}\right)=\hat{\varphi}_{i, A}\left(p_{0}, \vec{p}\right)=\hat{\varphi}_{i, A}^{p_{0}, \vec{p}_{A}}\left(\vec{p}_{A^{c}}\right) .
$$

Then,

$$
\begin{aligned}
\tilde{F}_{\alpha, N}\left(\varphi_{i, A}\right)= & \int F_{\alpha,|A|}\left(\varphi_{i, A}^{\vec{p}_{A^{c}}}\right) \mathrm{d} \vec{p}_{A^{c}} \\
& +\int\left\langle\varphi_{i, A}^{p_{0}, \vec{p}_{A}}\left|-\frac{1}{2} \sum_{i \in A^{c}} \Delta_{i}\right| \varphi_{i, A}^{p_{0}, \vec{p}_{A}}\right\rangle \mathrm{d} \vec{p}_{A} \mathrm{~d} p_{0} .
\end{aligned}
$$

Proof. We define $\xi_{j}$ and $\phi_{\mu}$ for some $\mu>0$ using the unique decomposition $\varphi_{i, A}=\phi_{\mu}+\sum_{j=1}^{N} G_{\mu} \xi_{j}$. Corollary 3.1 implies that $\xi_{j}=0$ for $j \in A^{c}$. Hence

$$
\begin{aligned}
\tilde{F}_{\alpha, N}\left(\varphi_{i, A}\right)= & \int \mathrm{d} \vec{p}_{A^{c}}\left[\int\left|\hat{\phi}_{\mu}\left(p_{0}, \vec{p}\right)\right|^{2}\left(\frac{1}{2 m} p_{0}^{2}+\frac{1}{2} \vec{p}^{2}+\mu\right) \mathrm{d} \vec{p}_{A} \mathrm{~d} p_{0}\right. \\
& -\mu \int\left|\hat{\varphi}_{i, A}\left(p_{0}, \vec{p}\right)\right|^{2} \mathrm{~d} \vec{p}_{A} \mathrm{~d} p_{0}
\end{aligned}
$$




$$
\begin{aligned}
& +\frac{2 m}{m+1} \alpha \sum_{i \in A} \int\left|\hat{\xi}_{i}\left(p_{i}, \hat{p}_{i}\right)\right|^{2} \mathrm{~d} \vec{p}_{A}+\sum_{i \in A} \int L_{\mu, N}\left(p_{i}, \hat{p}_{i}\right)\left|\hat{\xi}_{i}\left(p_{i}, \hat{p}_{i}\right)\right|^{2} \mathrm{~d} \vec{p}_{A} \\
& \left.-\sum_{\substack{i, j \in A \\
i \neq j}} \int \frac{\hat{\xi}_{i}^{*}\left(p_{0}+p_{i}, \hat{p}_{i}\right) \hat{\xi}_{j}\left(p_{0}+p_{j}, \hat{p}_{j}\right)}{\frac{1}{2 m} p_{0}^{2}+\frac{1}{2} \vec{p}^{2}+\mu} \mathrm{d} \vec{p}_{A} \mathrm{~d} p_{0}\right]
\end{aligned}
$$

Following the argumentation in the proof of Lemma 4.3, we see that the expression inside the integral over $\vec{p}_{A^{c}}$ is independent of $\mu$. In particular, this allows us to shift $\mu \rightarrow \mu-\vec{p}_{A^{c}}^{2} / 2$ for fixed $\vec{p}_{A^{c}}$, which gives

$$
\begin{aligned}
\tilde{F}_{\alpha, N}\left(\varphi_{i, A}\right) & =\int \mathrm{d} \vec{p}_{A^{c}}\left[\int\left|\hat{\phi}_{\mu-\vec{p}_{A_{c}}^{2} / 2}\left(p_{0}, \vec{p}\right)\right|^{2}\left(\frac{1}{2 m} p_{0}^{2}+\frac{1}{2} \vec{p}_{A}^{2}+\mu\right) \mathrm{d} \vec{p}_{A} \mathrm{~d} p_{0}\right. \\
& -\left(\mu-\frac{\vec{p}_{A^{c}}^{2}}{2}\right) \int\left|\hat{\varphi}_{i, A}\left(p_{0}, \vec{p}\right)\right|^{2} \mathrm{~d} \vec{p}_{A} \mathrm{~d} p_{0} \\
& +\frac{2 m}{m+1} \alpha \sum_{i \in A} \int\left|\hat{\xi}_{i}\left(p_{i}, \hat{p}_{i}\right)\right|^{2} \mathrm{~d} \vec{p}_{A}+\sum_{i \in A} \int L_{\mu,|A|}\left(p_{i}, \vec{p}_{A \backslash\{i\}}\right)\left|\hat{\xi}_{i}\left(p_{i}, \hat{p}_{i}\right)\right|^{2} \mathrm{~d} \vec{p}_{A} \\
& \left.-\sum_{\substack{i, j \in A \\
i \neq j}} \int \frac{\hat{\xi}_{i}^{*}\left(p_{0}+p_{i}, \hat{p}_{i}\right) \hat{\xi}_{j}\left(p_{0}+p_{j}, \hat{p}_{j}\right)}{\frac{1}{2 m} p_{0}^{2}+\frac{1}{2} \vec{p}_{A}^{2}+\mu} \mathrm{d} \vec{p}_{A} \mathrm{~d} p_{0}\right]
\end{aligned}
$$

where we used the fact that $L_{\mu-\vec{p}_{A^{c}}^{2} / 2, N}\left(p_{i}, \hat{p}_{i}\right)=L_{\mu,|A|}\left(p_{i}, \vec{p}_{A \backslash\{i\}}\right)$. The result then follows by noting that the Fourier transform of the regular part of $\varphi_{i, A}^{\vec{p}_{A^{c}}}$ for fixed $\vec{p}_{A^{c}}$ is equal to $\hat{\phi}_{\mu-\vec{p}_{A^{c}}^{2}}\left(\cdot, \vec{p}_{A^{c}}\right)$, and using the antisymmetry of $\varphi_{i, A}^{\vec{p}_{A^{c}}}$.

We can apply a similar decomposition also to the second term in (5.7). For simplicity, let

$$
W_{i}(x)=\frac{1}{2}\left(\left|\nabla V_{i}(x)\right|^{2}+\left|\nabla \tilde{V}_{i}(x)\right|^{2}\right) .
$$

Then, (5.7) and (5.9) imply that we can write

$$
F_{\alpha, N}\left(J_{i} \psi\right)=\sum_{A \subseteq\{1, \ldots, N\}}\left\|\varphi_{i, A}\right\|^{2}\left[\mathfrak{A}_{i, A}+\mathfrak{B}_{i, A}\right]
$$

where

$$
\mathfrak{A}_{i, A}=\left\|\varphi_{i, A}\right\|^{-2} \int\left(F_{\alpha,|A|}\left(\varphi_{i, A}^{\vec{p}_{A^{c}}}\right)-\left\langle\varphi_{i, A}^{\vec{p}_{A^{c}}}\left|\sum_{j \in A} W_{i}\left(x_{j}\right)\right| \varphi_{i, A}^{\vec{p}_{A^{c}}}\right\rangle\right) \mathrm{d} \vec{p}_{A^{c}}
$$

and

$$
\mathfrak{B}_{i, A}=\left\|\varphi_{i, A}\right\|^{-2} \int\left\langle\varphi_{i, A}^{p_{0}, \vec{p}_{A}}\left|\sum_{j \in A^{c}}\left(-\frac{1}{2} \Delta_{j}-W_{i}\left(x_{j}\right)\right)\right| \varphi_{i, A}^{p_{0}, \vec{p}_{A}}\right\rangle \mathrm{d} \vec{p}_{A} \mathrm{~d} p_{0} .
$$

To obtain a lower bound on $\mathfrak{A}_{i, A}$ we can use Theorem 4.1, and for the non-interacting part $\mathfrak{B}_{i, A}$ we use the following proposition. We recall that the energy $E_{n}^{D}$ on the box $\mathcal{C}_{L}=(0, L)^{3}$ was defined in the beginning of Sect. 2 as 
the ground-state energy of the non-interacting Hamiltonian $H_{0}^{n}$ with Dirichlet boundary conditions.

Proposition 5.1. For $n \in \mathbb{N}$, let $\phi \in H_{\text {as }}^{1}\left(\mathbb{R}^{3 n}\right)$ be supported in $(0, L)^{3 n}$, with $\|\phi\|_{2}=1$, and let $1 \leq i \leq M$. Then,

$$
\sum_{j=1}^{n} \int\left(\frac{1}{2}\left|\nabla_{j} \phi\right|^{2}-W_{i}\left(x_{j}\right)|\phi|^{2}\right) \geq E_{n}^{D}-\text { const. }\left(\frac{n^{1 / 3}}{\ell L}+\ell^{-2}+\frac{n \ell}{L^{3}}\right) \text {. }
$$

Proof. The result follows in a straightforward way from Corollary A.1, which is an adaptation of the Lieb-Thirring inequality at positive density derived in [14]. We use that $\left|\operatorname{supp}\left(W_{i}\right)\right| \lesssim \ell^{3}$ and $\left\|W_{i}\right\|_{\infty} \lesssim \ell^{-2}$. This allows us to bound the right side of $(\mathrm{A} .54)$ as

$$
\int_{\mathcal{C}_{L}}\left(\frac{n^{1 / 3}}{L}\left|W_{i}\right|^{2}+\left|W_{i}\right|^{5 / 2}+\frac{n}{L^{3}}\left|W_{i}\right|\right) \lesssim \frac{n^{1 / 3}}{\ell L}+\ell^{-2}+\frac{n \ell}{L^{3}}
$$

from which the statement readily follows.

Since $\varphi_{i, A}^{p_{0}, \vec{p}_{A}}$ is an antisymmetric function supported in $\mathcal{C}_{L}^{\left|A^{c}\right|}$, Proposition 5.1 implies that

$$
\begin{aligned}
& \left\langle\varphi_{i, A}^{p_{0}, \vec{p}_{A}}\left|\sum_{j \in A^{c}}\left(-\frac{1}{2} \Delta_{j}-W_{i}\left(x_{j}\right)\right)\right| \varphi_{i, A}^{p_{0}, \vec{p}_{A}}\right\rangle \\
& \quad \geq\left(E_{\left|A^{c}\right|}^{D}-\mathrm{const.}\left(\bar{\rho}^{1 / 3} \ell^{-1}+\ell^{-2}+\bar{\rho} \ell\right)\right)\left\|\varphi_{i, A}^{p_{0}, \vec{p}_{A}}\right\|^{2}
\end{aligned}
$$

where we used $\left|A^{c}\right| \leq N$ in the error term. To minimize the error, we choose $\ell \sim$ $\bar{\rho}^{-1 / 3}$. The factor on the right side of (5.16) then equals $E_{N-|A|}^{D}-$ const. $\bar{\rho}^{2 / 3}$. Because of the condition that $L / \ell \in \mathbb{N}$ we cannot choose $\ell$ without restriction, but it is always possible to choose a value such that $\ell \sim \bar{\rho}^{-1 / 3}$. We define $e_{N}$ to be the $N$-th eigenvalue of the one-particle Dirichlet Laplacian on $\mathcal{C}_{L}=(0, L)^{3}$. Then, $E_{N-|A|}^{D} \geq E_{N}^{D}-|A| e_{N}$. Moreover, we can bound $e_{N} \lesssim \bar{\rho}^{2 / 3}$. In particular,

$$
\mathfrak{B}_{i, A} \geq E_{N}^{D}-\text { const. }(|A|+1) \bar{\rho}^{2 / 3} \text {. }
$$

We proceed with a lower bound on $\mathfrak{A}_{i, A}$. Theorem 4.1 can be used for a lower bound on $F_{\alpha,|A|}$ only if $|A|>N_{0}$, with $N_{0}$ defined in (4.2). In case that $|A| \leq 2 N_{0}$ we use bound (2.2) originating form [25] instead, which implies that

$$
F_{\alpha,|A|}\left(\varphi_{i, A}^{\vec{p}_{A^{c}}}\right) \gtrsim-\frac{\alpha_{-}^{2}}{(1-\Lambda(m))^{2}}\left\|\varphi_{i, A}^{\vec{p}_{A^{c}}}\right\|^{2}
$$

using $m \gtrsim 1$. In combination with $\left\|W_{i}\right\|_{\infty} \lesssim \bar{\rho}^{2 / 3}$, this gives the lower bound

$$
\mathfrak{A}_{i, A} \gtrsim-\frac{\alpha_{-}^{2}}{(1-\Lambda(m))^{2}}-|A| \bar{\rho}^{2 / 3}
$$

and hence

$$
\mathfrak{A}_{i, A}+\mathfrak{B}_{i, A} \geq E_{N}^{D}-\text { const. }\left(\frac{\alpha_{-}^{2}}{(1-\Lambda(m))^{2}}+\left(N_{0}+1\right) \bar{\rho}^{2 / 3}\right)
$$

in case $|A| \leq 2 N_{0}$. 
For $|A| \geq 2 N_{0}$, we use the bound in Theorem 4.1 on $F_{\alpha,|A|}\left(\varphi_{i, A}^{\vec{p}_{A^{c}}}\right)$. Since $\varphi_{i, A}^{\vec{p}_{A^{c}}}$ is an $|A|+1$-particle wavefunction supported in a cube of side length $\ell(1+2 \varepsilon)$, Theorem 4.1 implies that

$$
F_{\alpha,|A|}\left(\varphi_{i, A}^{\vec{p}_{A^{c}}}\right) \geq\left(\kappa \frac{|A|^{5 / 3}}{\ell^{2}(1+2 \varepsilon)^{2}}-U\right)\left\|\varphi_{i, A}^{\vec{p}_{A^{c}}}\right\|^{2}
$$

with

$$
U=\frac{1}{4 \pi^{4}} \frac{m+1}{2 m} \frac{\left[\alpha-c_{L} \ell^{-1}\right]_{-}^{2}}{\left(1-\kappa / c_{T}-\Lambda(m)\right)^{2}\left(1-2^{-2 / 9}\right)^{2}} .
$$

In combination with (5.19) and $\left\|W_{i}\right\|_{\infty} \lesssim \bar{\rho}^{2 / 3}$, this yields the bound

$$
\begin{aligned}
\mathfrak{A}_{i, A}+\mathfrak{B}_{i, A} & \geq E_{N}^{D}+\kappa \frac{|A|^{5 / 3}}{\ell^{2}(1+2 \varepsilon)^{2}}-\text { const. }(|A|+1) \bar{\rho}^{2 / 3}-U \\
& \geq E_{N}^{D}-U-\text { const. } \kappa^{-3 / 2} \bar{\rho}^{2 / 3}
\end{aligned}
$$

where we have minimized over $|A|$ in the last step and used that $\varepsilon \lesssim 1$ and $\ell \sim \bar{\rho}^{-1 / 3}$.

We are still free to choose $\kappa$ in such a way as to minimize the error terms. We shall choose $\kappa=c_{T} \nu(1-\Lambda(m))$ for some $0<\nu<1$ (e.g., $\nu=1 / 2$ ). Then, $N_{0} \lesssim(1-\Lambda(m))^{-9 / 2}$, and hence (5.22) and (5.25) together yield the bound

$$
\begin{aligned}
\mathfrak{A}_{i, A}+\mathfrak{B}_{i, A} & \geq E_{N}^{D}-\text { const. }\left(\frac{\left[\alpha-c_{L} \ell^{-1}\right]_{-}^{2}}{(1-\Lambda(m))^{2}}+\frac{\bar{\rho}^{2 / 3}}{(1-\Lambda(m))^{9 / 2}}\right) \\
& \geq E_{N}^{D}-\text { const. }\left(\frac{\alpha_{-}^{2}}{(1-\Lambda(m))^{2}}+\frac{\bar{\rho}^{2 / 3}}{(1-\Lambda(m))^{9 / 2}}\right)
\end{aligned}
$$

which is valid for all $A \subset\{1, \ldots, N\}$. In combination with (5.3), (5.4) and (5.13), this completes the proof of Theorem 2.1.

\section{Appendix A: Lieb-Thirring inequality in a box}

In this appendix, we will follow the analysis of [14] to show a positive density Lieb-Thirring inequality for a system of non-interacting fermions in a box with Dirichlet boundary conditions. When reformulated via a Legendre transformation as a bound on the difference between the ground-state energies with and without an external potential, we will see that this inequality in particular implies Proposition 5.1. The analysis proceeds exactly as in [14], with minor differences due to the fact that the eigenfunctions of the Dirichlet Laplacian do not have constant modulus, which requires some modifications (in particular in Eq. (A.31) et seq.).

Let $\mathcal{C}_{L}=[-L / 2, L / 2]^{3}$ be the cube in $\mathbb{R}^{3}$, and let $\Pi_{L, \mu}^{-}:=\mathbb{1}\left(-\Delta_{L}\right.$ $\leq \mu$ ), where $\Delta_{L}$ denotes the Dirichlet Laplacian on $\mathcal{C}_{L}$. For short we will just write $\Pi^{-}$for $\Pi_{L, \mu}^{-}$, and $\Pi^{+}=1-\Pi^{-}$. For a density matrix $\gamma$, we denote the corresponding density by $\rho_{\gamma}$. Of particular relevance for us is the density 
corresponding to $\Pi^{-}$, which we denote by $\rho_{0}$. Differently to the case of periodic boundary conditions (discussed in [14]), $\rho_{0}$ is not a constant and is given by

$$
\rho_{0}(x)=\sum_{\substack{p \in \pi \mathbb{N}^{3} / L \\ p^{2} \leq \mu}}\left|\phi_{p}(x)\right|^{2}
$$

where $\phi_{p}$ are the eigenvectors of $-\Delta_{L}$ to the eigenvalues $p^{2}$, i.e.,

$$
\phi_{p}(x)=\left(\frac{2}{L}\right)^{3 / 2} \prod_{j=1}^{3} \cos \left(p_{j} x_{j}\right)
$$

for $x=\left(x_{1}, x_{2}, x_{3}\right) \in \mathbb{R}^{3}$. Since the absolute value of each eigenvector is pointwise bounded by $(2 / L)^{3 / 2}$ we have

$$
\rho_{0}(x) \leq\left(\frac{2}{L}\right)^{3} \sum_{\substack{p \in \pi \mathbb{N}^{3} / L \\ p^{2} \leq \mu}} 1 \leq\left(\frac{2}{L}\right)^{3} \frac{4 \pi}{3} \frac{\mu^{3 / 2} L^{3}}{\pi^{3}}=\frac{2^{5} \mu^{3 / 2}}{3 \pi^{2}} .
$$

Remark. Since the lowest eigenvalue of $-\Delta_{L}$ equals $3 \pi^{2} L^{-2}$, the problem simplifies for $\mu<3 \pi^{2} L^{-2}$ since the projections $\Pi_{L, \mu}^{ \pm}$become trivial. In this case, we can simply apply the original Lieb-Thirring inequality [17] to obtain the desired bound. For our application, we shall need $\mu \gg L^{-2}$; hence, we shall restrict our attention to $\mu \geq 3 \pi^{2} L^{-2}$ in the following theorem.

For a real number $t$, we denote its positive part by $t_{+}$and its negative part by $t_{-}$. In particular, $t=t_{+}-t_{-}$.

Theorem A.1. Let $\mu \geq 3 \pi^{2} L^{-2}$. Let $Q$ be a self-adjoint operator of finite rank satisfying $-\Pi_{L, \mu}^{-} \leq Q \leq 1-\Pi_{L, \mu}^{-}$, with density $\rho_{Q}$. There exist positive constants $\tilde{K}$ and $\eta$ independent of $\mu, L$ and $Q$ such that

$$
\operatorname{tr}\left(-\Delta_{L}-\mu\right) Q \geq \tilde{K} \int_{\mathcal{C}_{L}} S\left(\left(\left|\rho_{Q}(x)\right|-\eta L^{-1} \mu\right)_{+}\right) \mathrm{d} x
$$

with

$$
S(\rho):=\left(\mu^{3 / 2}+\rho\right)^{5 / 3}-\mu^{5 / 2}-\frac{5}{3} \mu \rho .
$$

Remark. In [14], a similar result was proven for the Laplacian with periodic boundary conditions and we mostly follow that proof.

Remark. The crucial properties of the function $S$ are its positivity and the fact that $S(\rho)$ behaves like $\mu^{-1 / 2} \rho^{2}$ for small $\rho$ and like $\rho^{5 / 3}$ for large $\rho$. For technical reasons, it will also be convenient that $S$ is convex.

Essential for the proof will be to separate a given $Q$ into $Q=\left(\Pi^{+}+\right.$ $\left.\Pi^{-}\right) Q\left(\Pi^{+}+\Pi^{-}\right)=: Q^{++}+Q^{+-}+Q^{-+}+Q^{--}$. The densities associated to $Q^{ \pm \pm}$will be denoted by $\rho^{ \pm \pm}$. Before we proceed with the proof of the theorem, we show the following Lemma.

Lemma A.1. Assume $\Pi^{-} \leq Q \leq 1-\Pi^{-}$. Then,

$$
\operatorname{tr}\left(\left|-\Delta_{L}-\mu\right| Q^{2}\right) \leq \operatorname{tr}\left(-\Delta_{L}-\mu\right) Q .
$$


Proof. We claim that $Q^{2} \leq Q^{++}-Q^{--}$, which follows from the condition on $Q$. In fact,

$$
-\Pi^{-} \leq Q \leq 1-\Pi^{-} \Rightarrow 0 \leq Q+\Pi^{-} \leq 1 \Rightarrow\left(Q+\Pi^{-}\right)^{2} \leq Q+\Pi^{-} .
$$

Expanding the last inequality proves the claim. Hence,

$$
\begin{aligned}
\operatorname{tr}\left(\left|\Delta_{L}+\mu\right| Q^{2}\right) & \leq \operatorname{tr}\left(\left|\Delta_{L}+\mu\right| Q^{++}\right)-\operatorname{tr}\left(\left|\Delta_{L}+\mu\right| Q^{--}\right) \\
& =\operatorname{tr}\left(\left(-\Delta_{L}-\mu\right) Q^{++}\right)+\operatorname{tr}\left(\left(-\Delta_{L}-\mu\right) Q^{--}\right) \\
& =\operatorname{tr}\left(\left(-\Delta_{L}-\mu\right) Q\right) .
\end{aligned}
$$

Proof of Theorem A.1. We shall treat $Q^{ \pm \pm}$separately and combine the various terms at the end using the convexity of $S$.

Part 1. $Q^{++}, Q^{--}$

We shall follow the method introduced by Rumin in [27]. With the aid of the spectral projections $P_{e}:=\mathbb{1}\left(\left|\Delta_{L}+\mu\right| \geq e\right)$, we have the layer cake representation

$$
\left|\Delta_{L}+\mu\right|=\int_{0}^{\infty} P_{e} \mathrm{~d} e
$$

Let us assume that $\gamma$ is a smooth enough finite rank operator with $0 \leq \gamma \leq 1$. Then,

$$
\operatorname{tr}\left|\Delta_{L}+\mu\right| \gamma=\int_{0}^{\infty} \mathrm{d} e \operatorname{tr}\left(P_{e} \gamma P_{e}\right)=\int_{0}^{\infty} \mathrm{d} e \int_{\mathcal{C}_{L}} \rho_{e}(x) \mathrm{d} x
$$

where $\rho_{e}$ denotes the density of the finite rank operator $P_{e} \gamma P_{e}$. For a bounded measurable set $A$, we estimate

$$
\begin{aligned}
\int_{A} \rho_{e}(x) \mathrm{d} x & =\operatorname{tr}\left(\mathbb{1}_{A} P_{e} \gamma P_{e}\right)=\left\|\mathbb{1}_{A} P_{e} \gamma^{1 / 2}\right\|_{\mathfrak{S}_{2}}^{2} \\
& \geq\left(\left\|\mathbb{1}_{A} \gamma^{1 / 2}\right\|_{\mathfrak{S}_{2}}-\left\|\mathbb{1}_{A} P_{e}^{\perp} \gamma^{1 / 2}\right\|_{\mathfrak{S}_{2}}\right)_{+}^{2} \\
& =\left(\left(\int_{A} \rho_{\gamma}\right)^{1 / 2}-\left\|\mathbb{1}_{A} P_{e}^{\perp} \gamma^{1 / 2}\right\|_{\mathfrak{S}_{2}}\right)_{+}^{2}
\end{aligned}
$$

where $\rho_{\gamma}$ denotes the density of $\gamma$ and we used the triangle inequality for the Hilbert-Schmidt norm $\|\cdot\|_{\mathfrak{S}_{2}}$. Because $\|\gamma\| \leq 1$, we further get

$$
\left\|\mathbb{1}_{A} P_{e}^{\perp} \gamma^{1 / 2}\right\|_{\mathfrak{S}_{2}}^{2}=\operatorname{tr}\left(\mathbb{1}_{A} P_{e}^{\perp} \gamma P_{e}^{\perp} \mathbb{1}_{A}\right) \leq\left\|\mathbb{1}_{A} P_{e}^{\perp}\right\|_{\mathfrak{S}_{2}}^{2}\|\gamma\| \leq|A| f(e)
$$

with 


$$
\begin{aligned}
f(e) & :=\left(\frac{2}{L}\right)^{3} \sum_{\substack{p \in \pi \mathbb{N}^{3} / L \\
\left|p^{2}-\mu\right|<e}} 1=\left(\frac{2}{L}\right)^{3} \sum_{\substack{n \in \mathbb{N}^{3} / 2 \\
\left|\frac{4 \pi^{2}}{L^{2}} n^{2}-\mu\right|<e}} 1 \\
& =\left(\frac{2}{L}\right)^{3}\left[\left|\mathbb{N}^{3} / 2 \cap B\left(\frac{L}{2 \pi}(\mu+e)^{1 / 2}\right)\right|-\left|\mathbb{N}^{3} / 2 \cap \bar{B}\left(\frac{L}{2 \pi}(\mu-e)_{+}^{1 / 2}\right)\right|\right]
\end{aligned}
$$

where $B(R)$ denotes the centered open ball with radius $R$ and $\bar{B}(R)$ its closure. Here, we used

$$
\left\|\mathbb{1}_{A} P_{e}^{\perp}\right\|_{\mathfrak{S}_{2}}^{2}=\sum_{\substack{p \in \pi \mathbb{N}^{3} / L \\\left|p^{2}-\mu\right|<e}} \int_{A}\left|\phi_{p}(x)\right|^{2} \mathrm{~d} x \leq|A| \sum_{\substack{p \in \pi \mathbb{N}^{3} / L \\\left|p^{2}-\mu\right|<e}} \sup _{\substack{x \in A \\ \mid p^{2}}}\left|\phi_{p}(x)\right|^{2} \leq|A| f(e)
$$

where we bounded the eigenfunction $\phi_{p}$ of $-\Delta_{L}$ to the eigenvalue $p^{2}$ by $\left|\phi_{p}(x)\right| \leq(2 / L)^{3 / 2}$. Taking $A=B(R)+x$ with $R \rightarrow 0$, we obtain the pointwise bound

$$
\rho_{e}(x) \geq\left(\sqrt{\rho_{\gamma}(x)}-\sqrt{f(e)}\right)_{+}^{2} .
$$

Hence, we get

$$
\operatorname{tr}\left|\Delta_{L}+\mu\right| \gamma \geq \int_{\mathcal{C}_{L}} \mathrm{~d} x \int_{0}^{\infty} \mathrm{d} e\left(\sqrt{\rho_{\gamma}(x)}-\sqrt{f(e)}\right)_{+}^{2}=\int_{\mathcal{C}_{L}} R\left(\rho_{\gamma}(x)\right) \mathrm{d} x
$$

with

$$
R(\rho):=\int_{0}^{\infty}(\sqrt{\rho}-\sqrt{f(e)})_{+}^{2} \mathrm{~d} e .
$$

To obtain the desired result, we have to analyze $R(\rho)$ in more detail. In the following, we will use $C$ to denote a generic constant, whose value can change throughout the computation. Obviously

$$
|| \mathbb{N}^{3} / 2 \cap B(R)\left|-\frac{4 \pi}{3} R^{3}\right| \lesssim \max \left(1, R^{2}\right)
$$

and the same statement holds if one takes the closure $\bar{B}(R)$ instead of $B(R)$. For $0<x<1$ and $M>0$, (A.18) allows us to bound

$$
\begin{aligned}
& \left|\mathbb{N}^{3} / 2 \cap B\left(M(1+x)^{1 / 2}\right)\right|-\left|\mathbb{N}^{3} / 2 \cap \bar{B}\left(M(1-x)^{1 / 2}\right)\right| \\
& \leq \frac{4 \pi M^{3}}{3}\left((1+x)^{3 / 2}-(1-x)_{+}^{3 / 2}\right)+C \max \left(1, M^{2}\right) \\
& \lesssim M^{3} x+\max \left(1, M^{2}\right)
\end{aligned}
$$

where we used $(1+x)^{3 / 2}-(1-x)_{+}^{3 / 2} \lesssim x$. Applying (A.19) to $f(e)$ for $e / \mu<1$, we get

$$
f(e) \lesssim \mu^{1 / 2} e+\frac{\mu}{L}
$$


using that $\mu \gtrsim L^{-2}$ by assumption. For $e \geq \mu$, we get

$$
f(e)=\left(\frac{2}{L}\right)^{3}\left|\mathbb{N}^{3} / 2 \cap B\left(\frac{L}{2 \pi}(\mu+e)^{1 / 2}\right)\right| \leq \frac{C}{L^{3}}\left(L^{3}(\mu+e)^{3 / 2}\right) \leq C e^{3 / 2} .
$$

Combining both statements, we have thus shown that

$$
f(e) \leq C\left(\frac{\mu}{L}+\mu^{1 / 2} e \mathbb{1}(e \leq \mu)+e^{3 / 2} \mathbb{1}(e>\mu)\right)=u+g(e)
$$

with

$$
g(e):=C e \max \left(\mu^{1 / 2}, e^{1 / 2}\right), \quad u:=C \frac{\mu}{L} .
$$

Using the explicit form of $g$, one readily checks that

$$
\begin{aligned}
R(\rho) & =\int_{0}^{\infty}(\sqrt{\rho}-\sqrt{f(e)})_{+}^{2} \mathrm{~d} e \\
& \geq \int_{0}^{\infty}(\sqrt{\rho}-\sqrt{u}-\sqrt{g(e)})_{+}^{2} \mathrm{~d} e \gtrsim S\left((\rho-2 u)_{+}\right),
\end{aligned}
$$

where we have also used that $(\sqrt{\rho}-\sqrt{u})_{+}^{2} \geq \frac{1}{2}(\rho-2 u)_{+}$. In combination with (A.16), this shows that

$$
\operatorname{tr}\left|-\Delta_{L}-\mu\right| \gamma \gtrsim \int_{\mathcal{C}_{L}} S\left(\left(\rho_{\gamma}(x)-C L^{-1} \mu\right)_{+}\right) \mathrm{d} x .
$$

We apply this for $\gamma=Q^{++}$and $\gamma=-Q^{--}$and obtain

$$
\operatorname{tr}\left(-\Delta_{L}-\mu\right) Q^{ \pm \pm} \gtrsim \int_{\mathcal{C}_{L}} S\left(\left(\left|\rho^{ \pm \pm}(x)\right|-C L^{-1} \mu\right)_{+}\right) \mathrm{d} x .
$$

Part 2. $Q^{+-}, Q^{-+}$

In the next step, we want to prove bounds for $Q^{+-}$and $Q^{-+}$. We introduce

$$
\begin{array}{ll}
\Pi_{0}^{+}=\mathbb{1}\left(\mu<-\Delta_{L}<\mu+\sqrt{\mu} / L\right), & \Pi_{0}^{-}=\mathbb{1}\left(\mu-\sqrt{\mu} / L \leq-\Delta_{L} \leq \mu\right) \\
\Pi_{1}^{+}=\mathbb{1}\left(\mu+\sqrt{\mu} / L \leq-\Delta_{L}\right), & \Pi_{1}^{-}=\mathbb{1}\left(-\Delta_{L}<\mu-\sqrt{\mu} / L\right)
\end{array}
$$

and split $Q^{+-}=\left(\Pi_{0}^{+}+\Pi_{1}^{+}\right) Q\left(\Pi_{0}^{-}+\Pi_{1}^{-}\right)=Q_{00}^{+-}+Q_{10}^{+-}+Q_{01}^{+-}+Q_{11}^{+-}$. The following three parts of the proof will treat these terms. We start with $Q_{00}^{ \pm}$.

Part 3. $Q_{00}^{+-}$

The density of $Q_{00}^{+-}$is equal to

$$
\rho_{00}^{+-}(x)=\sum_{\substack{k \in(\pi \mathbb{N} / L)^{3} \\ \mu<k^{2}<\mu+\sqrt{\mu} / L}} \sum_{\substack{j \in(\pi \mathbb{N} / L)^{3} \\ \mu-\sqrt{\mu} / L \leq j^{2} \leq \mu}}\left\langle\phi_{k} \mid Q \phi_{j}\right\rangle \phi_{k}(x) \phi_{j}(x) .
$$

Using $\|Q\| \leq 1$, we can bound this as

$$
\left.\left|\rho_{00}^{+-}(x)\right| \leq \sum_{\substack{k \in(\pi \mathbb{N} / L)^{3} \\ \mu<k^{2}<\mu+\sqrt{\mu} / L}}\left|\phi_{k}(x)\right|^{2}\right)^{1 / 2}\left(\sum_{\substack{j \in(\pi \mathbb{N} / L)^{3} \\ \mu-\sqrt{\mu} / L \leq j^{2} \leq \mu}}\left|\phi_{j}(x)\right|^{2}\right)^{1 / 2}
$$




$$
\begin{aligned}
& \leq\left(\frac{2}{L}\right)^{3} \sqrt{\left|\left\{\mu \leq k^{2} \leq \mu+\sqrt{\mu} / L\right\}\right|} \sqrt{\left|\left\{\mu-\sqrt{\mu} / L \leq j^{2} \leq \mu\right\}\right|} \\
& \leq C \frac{\mu}{L}
\end{aligned}
$$

where we applied (A.19) in the last step.

Part 4. $Q_{10}^{+-}, Q_{01}^{+-}$

Next we will bound $\rho_{10}^{+-}$. For a general function $W$ (viewed as a multiplication operator), we have

$$
\begin{aligned}
\left|\operatorname{tr}\left(W Q_{10}^{+-}\right)\right| & =\left|\operatorname{tr}\left(\Pi_{0}^{-} W \frac{\Pi_{1}^{+}}{\left|-\Delta_{L}-\mu\right|^{1 / 2}}\left|-\Delta_{L}-\mu\right|^{1 / 2} Q\right)\right| \\
& \leq \sqrt{\operatorname{tr}\left|-\Delta_{L}-\mu\right| Q^{2}}\left|\Pi_{0}^{-} W \frac{\Pi_{1}^{+}}{\left|-\Delta_{L}-\mu\right|^{1 / 2}}\right|_{\mathfrak{S}^{2}}
\end{aligned}
$$

To bound the first factor, we can use Lemma A.1. For the second term, we need to use the specific form of the eigenfunctions of the Dirichlet Laplacian. Using (A.2), we get

$$
\begin{aligned}
\left|\left\langle\phi_{p} \mid W \phi_{q}\right\rangle\right|^{2} & =\left(\frac{1}{2 L}\right)^{6}\left|\sum_{A, B \in\{1,-1\}^{3}} \hat{W}\left(\left(A_{j} p_{j}\right)_{j}-\left(B_{j} q_{j}\right)_{j}\right)\right|^{2} \\
& \lesssim L^{-6} \sum_{A, B \in\{1,-1\}^{3}}\left|\hat{W}\left(\left(A_{j} p_{j}\right)_{j}-\left(B_{j} q_{j}\right)_{j}\right)\right|^{2}
\end{aligned}
$$

where $\left(A_{j} p_{j}\right)_{j}$ and $\left(B_{j} q_{j}\right)_{j}$ denote the vectors obtained by component-wise multiplication. Hence,

$$
\begin{aligned}
& \left\|\Pi_{0}^{-} W \frac{\Pi_{1}^{+}}{\left|-\Delta_{L}-\mu\right|^{1 / 2}}\right\|_{\mathfrak{S}^{2}}^{2} \\
& =\sum_{\substack{p, q \in(\pi \mathbb{N} / L)^{3} \\
\mu-\sqrt{\mu} / L \leq p^{2} \leq \mu \\
q^{2}>\mu+\sqrt{\mu} / L}} \frac{\left|\left\langle\phi_{p} \mid W \phi_{q}\right\rangle\right|^{2}}{q^{2}-\mu} \leq \frac{L}{\sqrt{\mu}} \sum_{\substack{p, q \in(\pi \mathbb{N} / L)^{3} \\
\mu-\sqrt{\mu} / L \leq p^{2} \leq \mu \\
q^{2}>\mu+\sqrt{\mu} / L}}\left|\left\langle\phi_{p} \mid W \phi_{q}\right\rangle\right|^{2} \\
& \lesssim \frac{1}{L^{6}} \frac{L}{\sqrt{\mu}} \sum_{\substack{p, q \in(\pi(\mathbb{Z} \backslash\{0\}) / L)^{3} \\
\mu-\sqrt{\mu} / L \leq p^{2} \leq \mu \\
q^{2}>\mu+\sqrt{\mu} / L}}|\hat{W}(p-q)|^{2} \\
& \lesssim \frac{1}{L^{6}} \frac{L}{\sqrt{\mu}} \sum_{q \in(\pi(\mathbb{Z} \backslash\{0\}) / L)^{3}}|\hat{W}(q)|^{2} \sum_{\mu-\sqrt{\mu} / L \leq p^{2} \leq \mu} 1 \lesssim \sqrt{\mu}\|W\|_{2}^{2} .
\end{aligned}
$$

The sum of (A.31) is included in the second line of the previous calculation by extending the sum over $p, q \in \mathbb{N}^{3}$ to $p, q \in(\mathbb{Z} \backslash\{0\})^{3}$, and we have again used (A.19) in the last step. 
Choosing for $W=\left(\rho_{10}^{+-}\right)^{*}$, we thus get from (A.30)

$$
\int_{\mathcal{C}_{L}}\left|\rho_{10}^{+-}\right|^{2} \leq C \mu^{1 / 2} \operatorname{tr}\left(-\Delta_{L}-\mu\right) Q
$$

In a similar way, we can treat $\rho_{01}^{+-}$with the result that also

$$
\int_{\mathcal{C}_{L}}\left|\rho_{01}^{+-}\right|^{2} \leq C \mu^{1 / 2} \operatorname{tr}\left(-\Delta_{L}-\mu\right) Q \text {. }
$$

Part 5. $Q_{11}^{+-}$

Similarly to above we again introduce a multiplication operator $W$, and estimate

$$
\begin{aligned}
& \left|\operatorname{tr}\left(W \Pi_{1}^{+} Q \Pi_{1}^{-}\right)\right| \\
& \quad \leq\left\|\frac{\Pi_{1}^{+}}{\left|\Delta_{L}+\mu\right|^{1 / 4}} W \frac{\Pi_{1}^{-}}{\left|\Delta_{L}+\mu\right|^{1 / 4}}\right\|_{\mathfrak{S}^{2}}\left\|\left|\Delta_{L}+\mu\right|^{1 / 4} Q\left|\Delta_{L}+\mu\right|^{1 / 4}\right\|_{\mathfrak{S}^{2}} .
\end{aligned}
$$

The second factor we bound by

$$
\left\|\left|\Delta_{L}+\mu\right|^{1 / 4} Q\left|\Delta_{L}+\mu\right|^{1 / 4}\right\|_{\mathfrak{S}^{2}} \leq\left\|\left|\Delta_{L}+\mu\right|^{1 / 2} Q\right\|_{\mathfrak{S}^{2}}=\operatorname{tr}\left(\left|\Delta_{L}+\mu\right| Q^{2}\right)^{1 / 2}
$$

and Lemma A.1. For the first one, we have

$$
\begin{aligned}
& \left\|\frac{\Pi_{1}^{+}}{\left|\Delta_{L}+\mu\right|^{1 / 4}} W \frac{\Pi_{1}^{-}}{\left|\Delta_{L}+\mu\right|^{1 / 4}}\right\|_{\mathfrak{S}^{2}}^{2}=\sum_{\substack{p, q \in\left(\pi \mathbb{N}^{3} / L\right) \\
p^{2}>\mu+\sqrt{\mu} / L \\
q^{2}<\mu-\sqrt{\mu} / L}} \frac{\left|\left\langle\phi_{p} \mid W \phi_{q}\right\rangle\right|^{2}}{\left(\mu-q^{2}\right)^{1 / 2}\left(p^{2}-\mu\right)^{1 / 2}} \\
& \leq \frac{C}{L^{6}} \sum_{\substack{p, q \in\left(\pi \mathbb{Z}^{3} / L\right) \\
p^{2}>\mu+\sqrt{\mu} / L}}^{q^{2}<\mu-\sqrt{\mu} / L} \\
& \quad \frac{C}{L^{3}} \sum_{k \in\left(\pi \mathbb{Z}^{3} / L\right)} \Phi(k)|\hat{W}(q-p)|^{2}
\end{aligned}
$$

with

$$
\Phi(k)=\frac{1}{L^{3}} \sum_{\substack{q \in\left(\pi \mathbb{Z}^{3} / L\right) \\(q-k)^{2}>\mu+\sqrt{\mu} / L \\ q^{2}<\mu-\sqrt{\mu} / L}} \frac{1}{\left(\mu-q^{2}\right)^{1 / 2}\left((q-k)^{2}-\mu\right)^{1 / 2}} .
$$

In [14, Proof of Theorem 5.1], it was shown that $\sup _{k} \Phi(k) \lesssim \mu^{1 / 2}$ for $\mu \gtrsim L^{-2}$. Hence, the choice $W=\left(\rho_{11}^{+-}\right)^{*}$ yields

$$
\int_{\mathcal{C}_{L}}\left|\rho_{11}^{+-}\right|^{2} \lesssim \mu^{1 / 2} \operatorname{tr}\left(\left(-\Delta_{L}-\mu\right) Q\right) .
$$

Part 6. Combining the above estimates 
By combining (A.34) and (A.39), we obtain

$$
\mu^{-1 / 2} \int_{\mathcal{C}_{L}}\left|\rho^{+-}-\rho_{00}^{+-}\right|^{2} \leq C \operatorname{tr}\left(-\Delta_{L}-\mu\right) Q .
$$

Using that $\left|\rho_{00}^{+-}\right| \leq C \mu / L$, as shown in (A.29), this further implies that

$$
\mu^{-1 / 2} \int_{\mathcal{C}_{L}}\left(\left|\rho^{+-}\right|-C \mu / L\right)_{+}^{2} \leq C \operatorname{tr}\left(-\Delta_{L}-\mu\right) Q .
$$

The integrand in the left side is bounded from below by $C S\left(\left(\left|\rho^{+-}\right|-C \mu / L\right)_{+}\right)$; hence,

$$
\int_{\mathcal{C}_{L}} S\left(\left(\left|\rho^{+-}\right|-C \mu / L\right)_{+}\right) \leq C \operatorname{tr}\left(-\Delta_{L}-\mu\right) Q .
$$

Since $\left|\rho^{+-}\right|=\left|\rho^{-+}\right|$, the same bound holds for $\rho^{-+}$as well. Combining (A.26) and (A.42) and using the convexity of $S$, we get

$$
\begin{aligned}
\operatorname{tr}\left(-\Delta_{L}-\mu\right) Q & \gtrsim \int_{\mathcal{C}_{L}} S\left(\frac{\left(\left|\rho^{++}\right|+\left|\rho^{--}\right|+\left|\rho^{+-}\right|+\left|\rho^{-+}\right|-C \mu / L\right)_{+}}{4}\right) \\
& \geq \int_{\mathcal{C}_{L}} S\left(\frac{\left(\left|\rho_{Q}\right|-C \mu / L\right)_{+}}{4}\right) \gtrsim \int_{\mathcal{C}_{L}} S\left(\left(\left|\rho_{Q}\right|-C \mu / L\right)_{+}\right) .
\end{aligned}
$$

This completes the proof of Theorem A.1.

By taking a Legendre transform, the result above implies that following potential version of the Lieb-Thirring inequality.

Theorem A.2. Assume that $V$ is a real-valued function in $L^{5 / 2}\left([-L / 2,-L / 2]^{3}\right)$, and $\mu \geq 3 \pi^{2} L^{-2}$. Then, we have

$$
\begin{aligned}
0 & \geq-\operatorname{tr}\left(-\Delta_{L}+V-\mu\right)_{-}+\operatorname{tr}\left(-\Delta_{L}-\mu\right)_{-}-\int_{\mathcal{C}_{L}} \rho_{0} V \\
& \geq-K \int_{\mathcal{C}_{L}}\left(\mu^{1 / 2}|V|^{2}+|V|^{5 / 2}+L^{-1} \mu|V|\right)
\end{aligned}
$$

with $K>0$ independent of $L, \mu$ and $V$.

Remark. In case that $\mu<3 \pi^{2} L^{-2}$ we have $-\Delta_{L}-\mu>0$, and therefore $\operatorname{tr}\left(-\Delta_{L}-\mu\right)_{-}=0$ and also $\rho_{0}=0$. One can thus obtain a lower bound using the standard Lieb-Thirring inequality [17] applied to a potential $V-\mu$ in this case.

Proof. We start with the identity

$$
-\operatorname{tr}(A+B)_{-}=\inf _{0 \leq \gamma \leq 1} \operatorname{tr}(A+B) \gamma
$$

for Hermitian matrices $A$ and $B$, where an optimizer is clearly $\mathbb{1}(A+B \leq 0)$. With $P^{-}=\mathbb{1}(A \leq 0)$ and $Q=\gamma-P^{-},($A.45) reads

$$
-\operatorname{tr}(A+B)_{-}=\inf _{-P^{-} \leq Q \leq 1-P^{-}} \operatorname{tr}(A+B) Q+\operatorname{tr}(A+B) P^{-} .
$$


Defining $P_{B}^{-}=\mathbb{1}(A+B \leq 0)$, we equivalently get

$$
\operatorname{tr}(A+B)\left(P_{B}^{-}-P^{-}\right)=\inf _{-P^{-} \leq Q \leq 1-P^{-}} \operatorname{tr}(A+B) Q .
$$

This equality can be extended to allow $A=-\Delta-\mu$ and $B=V$ (see [14, Theorem 4.1]). Using this and applying Theorem A.1, we get

$$
\begin{array}{r}
\operatorname{tr}\left(-\Delta_{L}-\mu\right)_{-}-\operatorname{tr}\left(-\Delta_{L}+V-\mu\right)_{-}-\int_{\mathcal{C}_{L}} \rho_{0} V \\
\geq \inf _{\rho}\left(\tilde{K} \int_{\mathcal{C}_{L}} S\left(\left(|\rho|-\eta L^{-1} \mu\right)_{+}\right)+\int_{\mathcal{C}_{L}} V \rho\right) \\
\quad \geq \inf _{\rho \geq 0}\left(\tilde{K} \int_{\mathcal{C}_{L}} S\left(\left(\rho-\eta L^{-1} \mu\right)_{+}\right)-\int_{\mathcal{C}_{L}}|V| \rho\right)
\end{array}
$$

where the infimum in the first line is over functions $\rho: \mathbb{R}^{3} \rightarrow \mathbb{R}$, while in the second we can restrict to nonnegative functions $\rho$. We can pull the infimum inside the integral for a lower bound. Clearly, we can assume that $\rho \geq \eta L^{-1} \mu$. Introducing $\gamma=\rho-\eta L^{-1} \mu$, we have

$$
\begin{aligned}
& \inf _{\gamma \geq 0}\left(\tilde{K} S(\gamma)-|V| \gamma-\eta L^{-1} \mu|V|\right) \\
& \quad=\tilde{K}\left(\frac{2}{3} \mu^{5 / 2}+\tilde{K}^{-1}|V| \mu^{3 / 2}-\frac{2}{3}\left(\mu+\tilde{K}^{-1} \frac{3|V|}{5}\right)^{5 / 2}\right)-\eta L^{-1} \mu|V| .
\end{aligned}
$$

Using that

$$
x^{5 / 2}+\frac{5}{2} x^{3 / 2} y-(x+y)^{5 / 2} \geq-\frac{15 \sqrt{x} y^{2}}{8}-y^{5 / 2}
$$

for $x=\mu$ and $y=3 \tilde{K}^{-1}|V| / 5$ gives the bound

$$
\text { (A.49) } \gtrsim-\mu^{1 / 2}|V|^{2}-|V|^{5 / 2}-L^{-1} \mu|V| \text {. }
$$

Plugging this into (A.48) proves the Theorem.

We apply the above theorem for a potential $V \in L^{5 / 2}\left(\mathcal{C}_{L}\right)$ with $V \leq 0$, choosing $\mu$ as $e_{N}$, the $N$ th eigenvalue of the Dirichlet Laplacian $-\Delta_{L}$. In particular, $\mu \geq e_{1}=3 \pi^{2} L^{-2}$ which allows us to use Theorem A.2. The groundstate energy $E_{N}^{D}$ for $N$ non-interacting particles confined to $\mathcal{C}_{L}$ was defined in the beginning of Sect. 2 and can be written as $E_{N}^{D}=\sum_{i=1}^{N} e_{i}$.

We denote by $e_{k}^{V}$ the $k$ th eigenvalue of $-\Delta_{L}+V$, and by $E_{N}^{V, D}$ the sum of the $N$ lowest eigenvalues of $-\Delta_{L}+V$, i.e., $E_{N}^{V, D}=\sum_{i=1}^{N} e_{i}^{V}$. Theorem A.2 implies that

$$
\operatorname{tr}\left(-\Delta_{L}-\mu\right)_{-}=-E_{N}^{D}+N e_{N} \geq \operatorname{tr}\left(-\Delta_{L}+V-e_{N}\right)_{-}-R \geq-E_{N}^{V, D}+N e_{N}-R
$$

with

$$
R=\text { const. } \int_{\mathcal{C}_{L}}\left(\mu^{1 / 2}|V|^{2}+|V|^{5 / 2}+L^{-1} \mu|V|\right)-\int_{\mathcal{C}_{L}} \rho_{0} V .
$$


We used that since $V \leq 0$ the operator $-\Delta_{L}+V-e_{N}$ has at least $N$ nonpositive eigenvalues, and therefore we can get a lower bound on the trace of its negative part by summing only the first $N$ of them.

From the above calculation, together with $\rho_{0} \lesssim \mu^{3 / 2}$ and $\mu=e_{N} \lesssim$ $N^{2 / 3} / L^{2}$, we deduce the following corollary.

Corollary A.1. Let $V \in L^{5 / 2}\left(\mathcal{C}_{L}\right)$ with $V \leq 0$ and let $E_{N}^{D}$ denote the groundstate energy of $N$ non-interacting fermions confined to $\mathcal{C}_{L}$. With $E_{N}^{V, D}$, we denote the ground-state energy of the corresponding Hamiltonian with external potential $V$. Then,

$$
E_{N}^{D}-E_{N}^{V, D} \lesssim \int_{\mathcal{C}_{L}}\left(\frac{N^{1 / 3}}{L}|V|^{2}+|V|^{5 / 2}+\frac{N}{L^{3}}|V|\right)
$$

\section{Acknowledgements}

Open access funding provided by Institute of Science and Technology (IST Austria). We would like to thank Ulrich Linden for many helpful discussions. Financial support by the European Research Council (ERC) under the European Union's Horizon 2020 research and innovation programme (Grant Agreement No. 694227), and by the Austrian Science Fund (FWF), Project Nr. P 27533-N27, is gratefully acknowledged.

Open Access. This article is distributed under the terms of the Creative Commons Attribution 4.0 International License (http://creativecommons.org/licenses/ by/4.0/), which permits unrestricted use, distribution, and reproduction in any medium, provided you give appropriate credit to the original author(s) and the source, provide a link to the Creative Commons license, and indicate if changes were made.

\section{References}

[1] Albeverio, S., Gesztesy, F., Høegh-Krohn, R., Holden, H.: Solvable Models in Quantum Mechanics, 2nd edn. American Mathematical Society, Providence (2004)

[2] Basti, G., Cacciapuoti, C., Finco, D., Teta, A.: The three-body problem in dimension one: from short-range to contact interactions. J. Math. Phys. 59, 072104 (2018)

[3] Becker, S., Michelangeli, A., Ottolini, A.: Spectral properties of the $2+1$ fermionic trimer with contact interactions. Preprint arXiv:1712.10209

[4] Bethe, H., Peierls, R.: Quantum theory of the diplon. Proc. R. Soc. Lond. Ser. A 148, 146-156 (1935)

[5] Bethe, H., Peierls, R.: The scattering of neutrons by protons. Proc. R. Soc. Lond. Ser. A 149, 176-183 (1935)

[6] Correggi, M., Dell'Antonio, G., Finco, D., Michelangeli, A., Teta, A.: Stability for a system of $n$ fermions plus a different particle with zero-range interactions. Rev. Math. Phys. 24, 1250017 (2012) 
[7] Correggi, M., Dell'Antonio, G., Finco, D., Michelangeli, A., Teta, A.: A class of Hamiltonians for a three-particle fermionic system at unitarity. Math. Phys. Anal. Geom. 18, 1-36 (2015)

[8] Correggi, M., Finco, D., Teta, A.: Energy lower bound for the unitary $n+1$ fermionic model. Eur. Phys. Lett. 111, 10003 (2015)

[9] Cycon, H.L., Froese, R.G., Kirsch, W., Simon, B.: Schrödinger Operators. Springer Texts and Monographs in Physics (1987)

[10] Dell'Antonio, G.F., Figari, R., Teta, A.: Hamiltonians for systems of $n$ particles interacting through point interactions. Ann. Inst. Henri Poincaré 60, 253-290 (1994)

[11] Dimock, J., Rajeev, S.G.: Multi-particle Schrödinger operators with point interactions in the plane. J. Phys. A: Math. Gen. 37, 9157-9173 (2004)

[12] Fermi, E.: Sul moto dei neutroni nelle sostanze idrogenate. Ric. Sci. 7, 13-52 (1936)

[13] Finco, D., Teta, A.: Remarks on the Hamiltonian for the Fermionic Unitary Gas model. Rep. Math. Phys. 69, 131-159 (2010)

[14] Frank, R.L., Lewin, M., Lieb, E.H., Seiringer, R.: A positive density analogue of the Lieb-Thirring inequality. Duke Math. J. 162, 435-495 (2013)

[15] Griesemer, M., Linden, U.: Stability of the two-dimensional Fermi polaron. Lett. Math. Phys. 108, 1837-1849 (2018)

[16] Griesemer, M., Linden, U.: Spectral theory of the Fermi polaron. Preprint arXiv:1805.07229

[17] Lieb, E. H., Thirring, W.: Inequalities for the moments of the eigenvalues of the Schrödinger Hamiltonian and their relation to Sobolev inequalities. Studies in Mathematical Physics, pp. 269-303, Princeton University Press, Princeton (1976)

[18] Linden, U.: Energy estimates for the two-dimensional Fermi polaron. Ph.D. thesis, University of Stuttgart (2017)

[19] Linden, U., Mitrouskas, D.: High Density Limit of the Fermi Polaron with Infinite Mass. Preprint, arXiv:1807.06365

[20] Massignan, P., Zaccanti, M., Bruun, G.M.: Polarons, dressed molecules and itinerant ferromagnetism in ultracold Fermi gases. Rep. Prog. Phys. 77, 034401 (2014)

[21] Minlos, R.: On point-like interaction between $n$ fermions and another particle. Moscow Math. J. 11, 113-127 (2011)

[22] Minlos, R.A.: On pointlike interaction between three particles: two fermions and another particle. ISRN Math. Phys. 230245 (2012)

[23] Minlos, R.A.: A system of three quantum particles with point-like interactions. Russ. Math. Surv. 69, 539-564 (2014)

[24] Minlos, R.A.: On point-like interaction of three particles: two fermions and another particle. II. Moscow Math. J. 14, 617-637 (2014)

[25] Moser, T., Seiringer, R.: Stability of a fermionic $N+1$ particle system with point interactions. Commun. Math. Phys. 356, 329-355 (2017)

[26] Moser, T., Seiringer, R.: Stability of the $2+2$ fermionic system with point interactions. Math. Phys. Anal. Geom. 21, Art. 19 (2018) 
[27] Rumin, M.: Balanced distribution-energy inequalities and related entropy bounds. Duke Math. J. 160, 567-597 (2011)

[28] Thomas, L.H.: The interaction between a neutron and a proton and the structure of $H^{3}$. Phys. Rev. 47, 903-909 (1935)

[29] Wigner, E.: Über die Streuung von Neutronen an Protonen. Z. Phys. 83, 253-258 (1933)

[30] Zwerger, W. (ed.): The BCS-BEC Crossover and the Unitary Fermi Gas. Springer Lecture Notes in Physics 836 (2012)

Thomas Moser and Robert Seiringer

IST Austria

Am Campus 1

3400 Klosterneuburg

Austria

e-mail: robert.seiringer@ist.ac.at

Thomas Moser

e-mail: thomas.moser@ist.ac.at

Communicated by Jan Derezinski.

Received: July 12, 2018.

Accepted: December 7, 2018. 